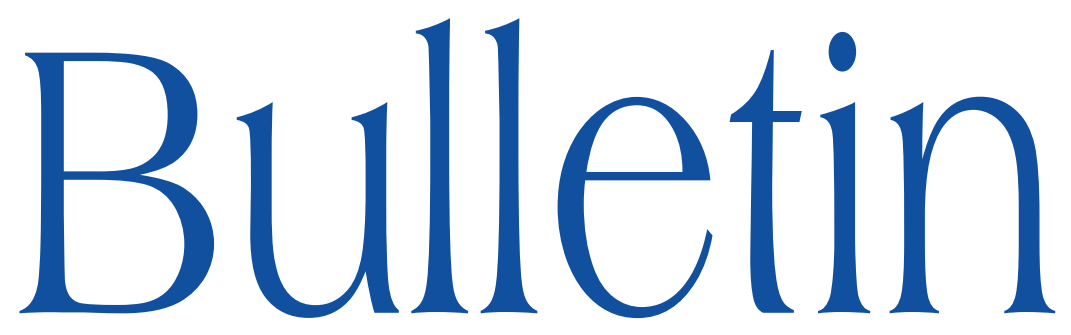

de la SOCIÉTÉ MATHÉMATIQUE DE FRANCE

\title{
GEOMETRIC STABILITY OF THE COTANGENT BUNDLE AND THE UNIVERSAL COVER OF A PROJECTIVE MANIFOLD
}

Frédéric Campana \& Thomas Peternell

Tome 139

Fascicule 1

2011 


\title{
GEOMETRIC STABILITY OF THE COTANGENT BUNDLE AND THE UNIVERSAL COVER OF A PROJECTIVE MANIFOLD
}

\author{
By Frédéric Campana \& Thomas Peternell \\ with an appendix by MATEI TOMA
}

\begin{abstract}
We first prove a strengthening of Miyaoka's generic semi-positivity theorem: the quotients of the tensor powers of the cotangent bundle of a non-uniruled complex projective manifold $X$ have a pseudo-effective (instead of generically nef) determinant. A first consequence is that $X$ is of general type if its cotangent bundle contains a subsheaf with 'big' determinant. Among other applications, we deduce that if the universal cover of $X$ is not covered by compact positive-dimensional analytic subsets, then $X$ is of general type if $\chi\left(O_{X}\right) \neq 0$. We finally show that if $L$ is a numerically trivial line bundle on $X$, and if $K_{X}+L$ is $\mathbb{Q}$-effective, then so is $K_{X}$ itself. The proof of this result rests on Simpson's work on jumping loci of numerically trivial line bundles, and Viehweg's cyclic covers. This last result is central, and has been recently extended, using the very same ingredients, to the case of log-canonical pairs.

Résumé (Stabilité géométrique du fibré cotangent et du recouvrement universel d'une variété projective)

Nous établissons tout d'abord un renforcement du théorème de semi-positivité de Miyaoka: le déterminant de tout quotient de toute puissance tensorielle du fibré cotangent d'une variété projective $X$ non-uniréglée est pseudo-effectif (au lieu de: génériquement nef). Une première conséquence est que $X$ est de type général si son fibré
\end{abstract}

Texte reçu le 28 juin 2007, révisé les 9 novembre 2007, 3 février 2009 et 2 juin 2009, accepté le 13 novembre 2009

Frédéric Campana, Département de Mathématiques, Université de Nancy, F-54506

Vandœuvre-les-Nancy, France • E-mail : frederic.campana@iecn.u-nancy.fr

Thomas Peternell, Mathematisches Institut, Universität Bayreuth, D-95440 Bayreuth, Germany - E-mail : thomas.peternell@uni-bayreuth.de

Matei Toma, Département de Mathématiques, Université de Nancy, F-54506 Vandœuvreles-Nancy, France • E-mail : toma@iecn.u-nancy.fr 2000 Mathematics Subject Classification. — 14J40, 32Q26, 32J27, 14E30.

Key words and phrases. — Bundle, pseudo-effective line bundle, Moishezon-Iitaka-'Kodaira' dimension, universal cover, uniruledness. 
cotangent a un sous-faisceau dont le déterminant est 'big'. Parmi diverses applications, nous montrons que si le revêtement universel de $X$ n'est pas recouvert par des sousensembles analytiques compacts de dimension strictement positive, alors $X$ est de type général si $\chi\left(O_{X}\right) \neq 0$.Nous montrons enfin que $K_{X}$ est $\mathbb{Q}$-effectif si $K_{X}+L$ l'est, pour un fibré en droites numériqiuement effectif $L$ sur $X$. La démonstration de ce résultat central repose sur les travaux de C. Simpson sur les lieux de Green-Lazarsfeld, et sur les revêtements cycliques de Viehweg. Ce résultat a été récemment étendu aux paires

'Log-canoniques' en utilisant les mêmes ingrédients.

\section{Introduction}

The aim of the present paper is to investigate birational positivity properties of the cotangent bundle of complex projective manifolds.

Our first result is the following sharpening of Miyaoka's uniruledness criterion:

Theorem 0.1. - Let $X$ be a projective manifold, $\left(\Omega_{X}^{1}\right)^{\otimes m} \rightarrow \&$ a torsion free coherent quotient for some $m \in \mathbb{N}$. Then $\operatorname{det} \&$ is pseudo-effective if $X$ is not uniruled.

Miyaoka's theorem asserts that the cotangent bundle of a projective manifold is "generically nef" unless the manifold is uniruled. A vector bundle $E$ is generically nef if $E \mid C$ is nef on the general curve cut out by very ample linear systems of sufficiently high degree. A line bundle $L$ is pseudo-effective if $c_{1}(L)$ lies in the closure of the Kähler cone. To sharpen generic nefness to pseudo-effectivity in the theorem, we use the characterization [2] of pseudoeffective line bundles by moving curves which are images of very ample curves above by birational morphisms. Our proof here is not entirely algebro-geometric (Mehta-Ramanathan no longer applies), and rests on analytic methods (see the appendix due to M. Toma).

A first consequence is:

ThEOREM 0.2. - Let $X$ be a projective manifold. Suppose that $\Omega_{X}^{p}$ contains for some $p$ a subsheaf whose determinant is big (i.e., has Kodaira dimension $n=\operatorname{dim} X)$. Then $K_{X}$ is big, i.e., $\kappa(X)=n$.

This uniruledness criterion has also other applications, e.g. one can prove that a variety admitting a section in a tensor power of the tangent bundle with a zero, must be uniruled.

Theorem 0.2 is actually a piece in a larger framework. To explain this, we consider subsheaves $\mathcal{F} \subset \Omega_{X}^{p}$ for some $p>0$. Then one can form $\kappa(\operatorname{det} \mathcal{F})$ and 
take the supremum over all $\mathcal{F}$. This gives a refined Kodaira dimension $\kappa^{+}(X)$, introduced in [3]. Conjecturally

$$
\kappa^{+}(X)=\kappa(X)
$$

unless $X$ is uniruled. Theorem 0.2 is nothing but this conjecture in case $\kappa^{+}(X)=\operatorname{dim} X$.

We shall prove the conjecture $(*)$ in several other cases. It is actually a consequence of the following more general conjecture, which moreover deals only with line bundles:

ConjeCture. - Suppose $X$ is a projective manifold, and suppose a decomposition

$$
N K_{X}=A+B
$$

with some positive integer $N$, an effective divisor $A$ (one may assume $A$ spanned) and a pseudo-effective line bundle $B$. Then

$$
\kappa(X) \geq \kappa(A) .
$$

The special case $A=\theta_{X}$ implies that $\kappa(X) \geq 0$ if $X$ is not uniruled, using the preceeding result, and the pseudo-effectiveness of $K_{X}$ when $X$ is not uniruled ([2]).

In another direction we establish the special case in which $B$ is numerically trivial:

Theorem 0.3. - Let $X$ be a projective complex manifold, and $L \in \operatorname{Pic}(X)$ be numerically trivial. Then:

1. $\kappa\left(X, K_{X}+L\right) \leq \kappa(X)$.

2. If $\kappa(X)=0$, and if $\kappa\left(X, K_{X}+L\right)=\kappa(X)$, then $L$ is a torsion element in the group $\operatorname{Pic}^{0}(X)$.

In particular, if $m K_{X}$ is numerically equivalent to an effective divisor, then $\kappa(X) \geq 0$.

This result permits, in particular, to handle numerically trivial line bundles in the study of the conjecture $C_{n, m}$ on irregular manifolds.

Another application of Theorem 0.2 is to the study of universal covers $\tilde{X}$ of complex projective $n$-dimensional manifolds $X$. The Shafarevich conjecture asserts that $\tilde{X}$ is holomorphically convex, i.e., admits a proper holomorphic map onto a Stein space. There are two extremal cases:

- either $\tilde{X}$ is compact and so $\pi_{1}(X)$ is finite or

- $\tilde{X}$ is a modification of a Stein space, hence through the general point of $\tilde{X}$ there is no positive-dimensional compact subvariety. 
This latter case happens in particular for $X$ a modification of an Abelian variety or a quotient of a bounded domain. It is conjectured (see [13], and [5] for the Kähler case) that $X$ should then admit a holomorphic submersion onto a variety of general type with Abelian varieties as fibres, after a suitable finite étale cover and birational modification. This follows up to dimension 3 from the solutions of the conjectures of the Minimal Model Program. We prove here a special case and a weaker statement in every dimension:

THEOREM 0.4. - Let $X$ be a normal $n$-dimensional projective variety with at most rational singularities.

(1) Suppose that the universal cover of $X$ is not covered by its positivedimensional compact subvarieties. Then $X$ is of general type if $\chi\left(\theta_{X}\right) \neq 0$.

(2) If $X$ has at most terminal singularities and $\tilde{X}$ does not contain any compact subvariety of positive dimension (eg. $X$ is Stein), then either $K_{X}$ is ample, or $K_{X}$ is nef, $K_{X}^{n}=0$, and $\chi\left(\theta_{X}\right)=0$.

This theorem is deduced from Theorem 0.2 above via the comparison theorem [3], which relates the geometric positivity of subsheaves in the cotangent bundle to the geometry of $\tilde{X}$.

Acknowledgement. - Our collaboration has been made possible by the priority program "Global methods in complex geometry" of the Deutsche Forschungsgemeinschaft, which we gratefully acknowledge.

We thank P. Eyssidieux, T. Eckl, J. Stix for pointing out a gap in the first version, and also C. Mourougane for his interesting observation on our previous Remark 3.6. We also thank the referee for his careful reading and for pointing out several inacuracies and mistakes in previous versions.

\section{Uniruledness Criteria}

Our main tool which is of independent interest, is a generalisation 1.4 of Miyaoka's uniruledness Criterion 1.2, which we recall first.

Definition 1.1. - Let $X$ be a complex projective $n$-dimensional manifold. $A$ vector bundle $E$ over $X$ is generically nef, if for all ample line bundles $H_{1}, \ldots, H_{n-1}$, for all $m_{i}$ sufficiently large and for general curves $C$ cut out by $m_{1} H_{1}, \ldots, m_{n-1} H_{n-1}$, the bundle $E \mid C$ is nef.

Miyaoka's criterion [17], with a short proof in [23], is now the following

THEOREM 1.2. - The cotangent bundle of a projective manifold is generically nef if $X$ is not uniruled.

TOME $139-2011-\mathrm{N}^{\circ} 1$ 
Via the theorem of Mehta-Ramanathan [16] and the uniruledness criterion of Miyaoka-Mori [19]. Theorem 1.2 is easily seen to be equivalent to the following statement:

If the $n$-dimensional projective manifold $X$ is not uniruled and $\Omega_{X}^{1} \rightarrow Q \rightarrow 0$ a torsion free quotient, then

$$
c_{1}(Q) \cdot H_{1} \cdots H_{n-1} \geq 0
$$

for all ample divisors $H_{i}$ on $X$.

Before stating the first generalization in 1.4 below, we need to introduce the notion of movable class of curves, generalising complete intersections curves.

We will denote by $\overline{M E}(X)$ the closed cone of (classes of) movable curves, as defined in [2]. This is the smallest closed cone containing all the classes of movable curves: a curve $C$ is movable if it belongs to a covering family $\left(C_{t}\right)_{t \in T}$ of curves which is to say that $T$ is irreducible and projective, the general $C_{t}$ is irreducible and the $C_{t}$ covers $X$.

One of the main results of [2] is that $\overline{M E}(X)$ is the closed convex cone generated by classes $\alpha$ of the form $\alpha=\pi_{*}\left(H_{1} \cap \cdots \cap H_{n-1}\right)$, with $\pi: X^{\prime} \rightarrow X$ a modification and $H_{j}$ very ample on $X^{\prime}$, see (1.8) below.

Let $\alpha \in \overline{M E}(X)$. The slope of a torsion free sheaf $\mathcal{E}$ of rank $r$ with respect to $\alpha$ is defined by

$$
\mu_{\alpha}(\mathcal{E})=\frac{c_{1}(\mathcal{E}) \cdot \alpha}{r}
$$

A torsion free sheaf is $\alpha$-semi-stable, if for all proper non-zero coherent subsheaves $\mathcal{F} \subset \mathcal{E}$ :

$$
\mu_{\alpha}(\mathcal{F}) \leq \mu_{\alpha}(\mathcal{E})
$$

The general properties of $\alpha$-slopes are very much parallel to the classical polarized case $\alpha=H_{1} \cdots H_{n-1}$ with ample line bundles $H_{i}$.

Proposition 1.3. - Let $X$ be a projective manifold and $\alpha \in \overline{M E}(X)$. Let $\mathcal{E}$ be a non-zero coherent torsion free sheaf on $X$. Then:

1. When $\mathcal{F}$ ranges over all nonzero proper coherent subsheaves of $\mathcal{E}$, the slope $\mu_{\alpha}(\mathcal{F})$ is bounded from above.

Let $\mu_{\alpha}^{\max }(\mathcal{E})$ be the maximum value, which is attained if $\alpha$ is a rational class.

2. If $\alpha$ is a rational class, there exists a unique largest subsheaf $\mathcal{E}^{\text {max }} \subset \mathcal{E}$ such that

$$
\mu_{\alpha}\left(\mathcal{E}^{\max }\right)=\mu_{\alpha}^{\max }(\mathcal{E}) .
$$

The quotient $\mathcal{E} / \mathcal{E}^{\max }$ is torsion free. 
3. Define inductively

$$
\mathcal{E}_{0}=\{0\} \subset \mathcal{E}_{1}=\mathcal{E}^{\max } \subset \cdots \subset \mathcal{E}_{s+1}=\mathcal{E}
$$

such that $\left(\mathcal{E}_{j+1} / \mathcal{E}_{j}\right)=\left(\varepsilon_{/} / \mathcal{E}_{j}\right)^{\max }$, for $j=0, \ldots, s$. This sequence is called the Harder-Narasimhan filtration of $\mathcal{E}$ relative to $\alpha$. We write

$$
\mu_{\alpha}^{\min }(\mathcal{E}):=\mu\left(\varepsilon / \mathcal{E}_{s}\right) .
$$

The quotients $\mathcal{E} / \mathcal{E}_{j}$ are the $\alpha$-semistable pieces of the $H N$-filtration of $\mathcal{E}$ relative to $\alpha$.

4. $\mu_{\alpha}\left(\mathcal{E}_{j+1} / \mathcal{E}_{j}\right)=\mu_{\alpha}^{\max }\left(\mathcal{E} / \mathcal{E}_{j}\right)>\mu_{\alpha}\left(\mathcal{E} / \mathcal{E}_{j+1}\right)$, for $j \geq 0$.

5. $\operatorname{Hom}\left(\mathcal{E}_{j}, \mathcal{E} / \mathcal{E}_{j}\right)=0$ for all $j \geq 0$, once $\mu_{\alpha}\left(\mathcal{E}_{j}\right) \geq 0$.

6. Let $\alpha \in \overline{M E}(X) \cap H^{2 n-2}(X, \mathbb{Q})$ with $n=\operatorname{dim} X$, and let $\mathcal{E}$ and $\mathcal{F}$ be $\alpha$-semi-stable torsion free sheaves on $X$. Then $\mathcal{E} \hat{\otimes} \mathcal{F}:=(\mathscr{E} \otimes \mathcal{F}) /$ tor is again $\alpha$-semi-stable.

7. $\operatorname{Hom}\left(\wedge^{2} \mathcal{E}_{j}, \mathcal{E} / \mathcal{E}_{j}\right)=0$ for all $j \geq 0$, if $\mu_{\alpha}\left(\mathcal{E}_{k}\right) \geq 0$ for all $k \leq j$.

Proof. - The proof of the first four statements is essentially the same as in the classical case of polarised varieties, see e.g. [20, p. 62]. Let us give a hint for the proof of (1): expressing $\mathcal{E}$ as a quotient of $V_{N}:=\Theta(m H)^{\oplus N}$ for arbitrary ample $H$ and suitable integers $m, N>0$, we are reduced to the case of $V_{N}$ for the first statement. This case is easily dealt with by induction on $N$, for fixed $m, H$. The second statement, for $\alpha$ rational, follows immediately from the fact that the $\alpha$-slopes are then rational numbers with uniformly bounded denominators.

The last two properties follow from property (4), and the fact (see also [23]) that $\operatorname{Hom}(\mathcal{E}, \mathcal{F})=0$ if $\mu_{\alpha}^{\min }(\mathcal{E})>\mu_{\alpha}^{\max }(\mathcal{F})$. Property (6) is more delicate, and proved in the appendix. For property (7), we proceed in the usual way (see [23]), using (6).

The first generalization of Theorem 1.2 is

THEOREM 1.4. - Let $X$ be a connected projective manifold and $\alpha \in \overline{M E}(X)$ of the form

$$
\alpha=\pi_{*}\left(H_{1} \cap \cdots \cap H_{n-1}\right)
$$

with $\pi: X^{\prime} \rightarrow X$ a modification and $H_{j}$ very ample on $X^{\prime}$. If there exists a torsion free quotient sheaf

$$
\Omega_{X}^{1} \rightarrow Q \rightarrow 0
$$

such that $c_{1}(Q) \cdot \alpha<0$, then $X$ is uniruled.

In other words, if $\left(C_{t}\right)$ is a covering family of curves which is the birational image of hyperplane sections with $c_{1}(Q) \cdot C_{t}<0$, then $X$ is uniruled. 
REMARK 1.1. - $\quad$ 1. We recall some notation used in (1.4). Let $\mathcal{F}$ be a coherent sheaf of rank $r$ on the connected manifold $X$. We define its determinant - a line bundle since $X$ is smooth - to be

$$
\operatorname{det} \mathcal{F}=\left(\bigwedge^{r} \mathcal{F}\right)^{* *}
$$

We also set $c_{1}(\mathcal{F})=c_{1}(\operatorname{det} \mathcal{F})$.

2. The last assumption in Theorem 1.4 cannot be weakened to assuming that, for generic $t \in T$, the bundle $\Omega_{X \mid C_{t}}^{1}$ is not nef (i.e., $\Omega_{X}^{1} \mid C_{t}$ has a quotient $Q_{t}$ such that $\operatorname{deg}\left(Q_{t}\right)<0$ ). See [2], Theorem 7.7.

3. The last assumption is however satisfied if, for generic $t \in T, \Omega_{X \mid C_{t}}^{1}$ is not nef, provided $C_{t}$ is an ample curve obtained as intersection of $(n-1)$ generic members of a sufficiently high multiple of some polarisation $H$ on $X$. This is a consequence of [16]. See [23].

Question 1.5. - Let $X$ be a projective manifold and $\pi: X^{\prime} \rightarrow X$ be a modification from another projective manifold $X^{\prime}$. Is $\pi^{*}\left(\Omega_{X}^{1}\right)$ generically nef if $X$ is not uniruled?

The problem is to show that the last assumption of 1.4 is satisfied, if $C_{t}=$ $\pi_{*}\left(C_{t}^{\prime}\right)$, where $C_{t}^{\prime}$ is a sufficiently ample curve on $X^{\prime}$, as in the preceding Remark 1.1 .

Proof of 1.4. - The proof follows the line of argumentation in [23], using the notion of Harder-Narasimhan filtration for $\alpha \in \overline{M E}(X)$. Observe that we cannot use [16] in our context.

So assume that $X$ is not uniruled. Then

$$
K_{X} \cdot \alpha \geq 0
$$

by [2], stated as Theorem 1.8 below. Hence $\Omega_{X}^{1}$ is not $\alpha$-semi-stable, since the kernel of

$$
\Omega_{X}^{1} \rightarrow Q \rightarrow 0
$$

destabilizes $\Omega_{X}^{1}$. Thus also its dual $T_{X}$ is not $\alpha$-semi-stable.

We now define

$$
\mathcal{F} \subset T_{X}
$$

to be the maximal destabilising subsheaf of $T_{X}$ relative to $\alpha$. Then Proposition 1.3(7) applies and we conclude that $\mathcal{F}$ is Lie closed.

We furthermore introduce $\mathscr{G}=T_{X} / \mathcal{F}$ and also fix an ample divisor $H$ on $X$ such that $T_{X}(H)$ is spanned.

Following the arguments in [23], we now reduce to char $p$ and want to prove that $\mathcal{F}_{p}$ is $p$-closed. To make the notations not too clumsy, the reduction mod 
$p$ will carry the same notation. So let $F: X_{p} \rightarrow X_{p}$ denote the absolute Frobenius; we need to prove that

$$
\operatorname{Hom}\left(F^{*}\left(\mathcal{F}_{p}\right), \mathscr{G}_{p}\right)=0 .
$$

Instead of restricting to curves as in [23] — which will not work in our situationwe first observe that [24, Prop.1] remains true with exactly the same proof in our situation (i.e., with $\alpha$ instead of an ample polarisation), since $\mathcal{F}$ is $\alpha$-semistable. By Theorem 5.7, the reduction $\bmod p, \mathcal{F}=\mathcal{F}_{p}$ remains $\alpha_{p}$-semistable for large $p$.

This gives the following. If

$$
0=\mathscr{G}_{1} \subset \mathscr{G}_{2} \subset \cdots \subset \mathscr{G}_{m}=F^{*}(\mathcal{F})
$$

is the HN-filtration of $F^{*}(\mathcal{F})$ relative to $\alpha$, then there are non-zero homomorphisms

$$
T_{X} \rightarrow \operatorname{Hom}\left(\mathscr{G}_{i}, F^{*}(\mathcal{F}) / \mathscr{G}_{i}\right)
$$

From this we derive for any index $i$ the existence of some index $j \geq i$ and a non-zero homomorphism

$$
T_{X} \rightarrow \operatorname{Hom}\left(\mathscr{G}_{i}, \mathscr{G}_{j+1} / \mathscr{G}_{j}\right)
$$

Consequently

$$
\mu_{\alpha}^{\min }\left(\mathscr{G}_{i}\right) \leq \mu_{\alpha}^{\max }\left(\left(\mathscr{G}_{j+1} / \mathscr{G}_{j}\right) \otimes \Omega_{X}^{1}\right) \leq \mu_{\alpha}^{\max }\left(\left(F^{*}(\mathcal{F}) / \mathscr{G}_{i}\right) \otimes \Omega_{X}^{1}\right) . \quad(++)
$$

We now follow the arguments of the first few lines of $[14,2.5]$ practically verbatim to show that

$$
\mu_{\alpha}^{\max }\left(F^{*}(\mathcal{F})\right)-\mu_{\alpha}^{\min }\left(F^{*}(\mathcal{F})\right)
$$

is bounded independently of $p$. Indeed we show

$$
\mu_{\alpha}^{\max }\left(F^{*}(\mathcal{F})\right)-\mu_{\alpha}^{\min }\left(F^{*}(\mathcal{F})\right) \leq(\operatorname{rk}(\mathcal{F})-1) H \cdot \alpha .
$$

Indeed, by the choice of $H$, the bundle $\Omega_{X}^{1}$ embeds into $\vartheta_{X}(X)^{\oplus N}$ for some positive $N$. Recalling the inequality $(++)$

$$
\mu_{\alpha}^{\min }\left(\mathscr{G}_{i}\right) \leq \mu_{\alpha}^{\max }\left(\left(F^{*}(\mathcal{F}) / \mathscr{G}_{i}\right) \otimes \Omega_{X}^{1}\right)
$$

we obtain

$$
\mu_{\alpha}^{\max }\left(\left(F^{*}(\mathcal{F}) / \mathscr{G}_{i}\right) \otimes \Omega_{X}^{1}\right) \leq \mu_{\alpha}^{\max }\left(\left(F^{*}(\mathcal{F}) / \mathscr{G}_{i}\right) \otimes \Theta_{X}(H)\right) .
$$

Summing up the inequalities as in [14, 2.5],

$$
\mu_{\alpha}\left(\mathscr{G}_{i} / \mathscr{G}_{i-1}\right) \leq \mu_{\alpha}\left(\mathscr{G}_{i+1} / \mathscr{G}_{i}\right)+H \cdot \alpha
$$

gives $(* *)$.

This implies the $p$-closedness of $\mathcal{F}$ by [23, 9.1.3.5]

Thus $\mathcal{F}$ is Lie closed and $p$-closed (and therefore $\mathcal{F}$ is a 1 -foliation in the terminology of [23]). 
Following further the argumentation in [23], we get a quotient map

$$
\rho: X \rightarrow Y=X / \mathcal{F} .
$$

We are now going to apply [18]. For this, we take the normalization $f: C \rightarrow X$ of some member $C_{t}$ (a covering family of curves representing the class $\alpha-$ these curves arise as images of complete intersection curves on some birational model). Consider the subspace $\operatorname{Hom}_{Y}(C, X)$ of $\operatorname{Hom}(C, X)$ consisting of maps $g: C \rightarrow X$ such that $\rho \circ g=\rho \circ f$. Then we have the basic inequality, proved in [18], Theorem 1:

$$
\operatorname{dim}_{[f]} \operatorname{Hom}_{Y}(C, X) \geq \chi\left(f^{* *}(\mathcal{F})\right) .
$$

Here

$$
f^{* *}(\mathcal{F})=f^{*}(\mathcal{F}) / \text { torsion }=\operatorname{Ker}\left(f^{*}(d \rho): f^{*}\left(T_{X}\right) \rightarrow g^{*}\left(T_{Y}\right)\right) .
$$

Combining $f$ with the geometric Frobenius on $C$, Riemann-Roch gives

$$
\chi\left(f^{* *}(\mathcal{F})\right)=p\left(c_{1}(\mathcal{F}) \cdot C\right)+(1-g(C)) \operatorname{rk}(\mathcal{F}) .
$$

Now we can proceed as in the classical case in [19] to obtain the claimed uniruledness.

We shall need the following generalization

THEOREM 1.6. - Let $X$ be a connected projective manifold, and $\alpha \in \overline{M E}(X)$ of the form

$$
\alpha=\pi_{*}\left(H_{1} \cap \cdots \cap H_{n-1}\right)
$$

with $\pi: X^{\prime} \rightarrow X$ a modification and $H_{j}$ very ample on $X^{\prime}$. If there exists a torsion free quotient sheaf

$$
\left(\Omega_{X}^{1}\right)^{\otimes m} \rightarrow Q \rightarrow 0
$$

for some $m \in \mathbb{N}$, such that $c_{1}(Q) \cdot \alpha<0$, then $X$ is uniruled.

Proof. - As in the proof of Theorem 1.4, $\left(\Omega_{X}^{1}\right)^{\otimes m}$ is not $\alpha$-semi-stable; let $\phi_{m}$ be the maximal destabilizing subsheaf. From our assumption

$$
\mu_{\alpha}^{\max }\left(\left(\Omega_{X}^{1}\right)^{\otimes m}\right)=\mu_{\alpha}\left(\phi_{m}\right)>\mu_{\alpha}\left(\left(\Omega_{X}^{1}\right)^{\otimes m}\right)>0 .
$$

Hence by Theorem $5.1 \Omega_{X}^{1}$ itself is not $\alpha$-semi-stable. Let $\phi_{1} \subset \Omega_{X}^{1}$ be the maximal destabilizing subsheaf with torsion free quotient $Q_{1}$. By Corollary 5.4, we obtain

$$
\mu_{\alpha}^{\max }\left(\Omega_{X}^{1}\right)=\mu_{\alpha}\left(\phi_{1}\right)>0
$$

Hence

$$
c_{1}\left(Q_{1}\right) \cdot \alpha<0,
$$

and $X$ is uniruled by Theorem 1.4 . 
Now we can strengthen the preceeding result, using [2] (and answering a question asked in that paper).

First recall that a line bundle $L$ on a projective manifold is called pseudoeffective iff $c_{1}(L)$ is in the closure of the cone generated by the (numerical equivalence classes of the) effective divisors on $X$.

We will need the following result from [2] which will also be crucial for Theorem 2.3.

TheOREM 1.7. - Let $X_{n}$ be a projective manifold of dimension $n$ and $L$ a line bundle on $X$. Then $L$ is pseudo-effective if and only if the following holds. Let $\pi: \hat{X} \rightarrow X$ be a birational map from a projective manifold $X$. Let $H_{1}, \ldots, H_{n-1}$ be very ample line bundles on $\hat{X}$. Then

$$
L \cdot \pi_{*}\left(H_{1} \cap \cdots \cap H_{n-1}\right) \geq 0 .
$$

Together with Theorem 1.7, this implies:

THEOREM 1.8. - Let $X$ be a projective manifold and suppose that $X$ is not uniruled. Let $Q$ be a torsion free quotient of $\left(\Omega_{X}^{1}\right)^{\otimes m}$ for some $m>0$. Then $\operatorname{det} Q$ is pseudo-effective.

Proof. - In order to show the pseudo-effectivity of $\operatorname{det} Q$, it suffices by (1.8) to verify the following.

Let $\pi: \tilde{X} \rightarrow X$ be birational from the projective manifold $\tilde{X}$. Let $H_{1}, \ldots, H_{n-1}$ be (very) ample on $\tilde{X}$. Then

$$
\operatorname{det} Q \cdot \pi_{*}\left(H_{1} \cap \cdots \cap H_{n-1}\right) \geq 0 .
$$

This is however verified by (1.7).

Now a pseudo-effective line bundle is nef on moving curves; here "moving" means that the deformations of the curve cover the variety. Actually by [2] the closed cone generated by numerical equivalence classes of movable curves coincides with the cone generated by classes of "strongly movable" curves. These are just the curves of the form $\pi(\hat{C})$, where $\pi: \hat{X} \rightarrow X$ is a modification, and $\hat{C} \subset \hat{X}$ is a generic intersection of very ample divisors $m_{i} H_{i}, 1 \leq i \leq n-1$ on $\hat{X}$. So we can state:

Corollary 1.9. - Let $X$ be a projective manifold and suppose that $X$ is not uniruled. Let $\left(C_{t}\right)_{t \in T}$ be an algebraic family of curves, parametrised by the irreducible projective variety $T$. Assume this family is covering (i.e.: the union of the $C_{t}$ 's is $X$, and its generic member is irreducible).

Let $\mathcal{F}$ be a torsion free quotient of $\left(\Omega_{X}^{1}\right)^{\otimes m}$ for some $m>0$. Then $c_{1}(\mathcal{F}) \cdot C_{t} \geq 0$. 
Corollary 1.10. - Let $X$ be a projective manifold and L a topologically trivial line bundle on $X$. Let $m$ be a positive integer and

$$
v \in H^{0}\left(T_{X}^{\otimes m} \otimes L\right)
$$

a section with zeroes in codimension 1. Then $X$ is uniruled.

More generally, suppose that $\mathcal{F} \subset T_{X}^{\otimes m}$ is a coherent subsheaf of rank $r$ such that $\operatorname{det} \mathcal{F}$ is pseudo-effective and that $\operatorname{det} \mathcal{F} \rightarrow \Lambda^{r}\left(T_{X}^{\otimes r}\right)$ has zeroes in codimension 1. Then $X$ is uniruled.

Proof. - Choose $p \in X$ such that $v(p)=0$. Let $\pi: \hat{X} \rightarrow X$ be the blow-up of $X$ at $p$. Assume that $X$ is not uniruled. Then, supposing that (1.6) has a positive answer, $\pi^{*}\left(\Omega_{X}^{1}\right)$ is generically nef. Hence if $\hat{C}$ is the curve cut out by sufficiently general very ample divisors, then $\pi^{*}\left(\Omega_{X}^{1}\right) \mid \hat{C}$ is nef. Thus $\Omega_{X}^{1} \mid C$ is nef, where $C=\pi(\hat{C})$. Now $\hat{C}$ meets the exceptional divisor of $\pi$ in a finite set, hence $p \in C$. In total, $\left(\Omega_{X}^{1}\right)^{\otimes m} \otimes L^{*} \mid C$ is nef, but its dual has a section with zeroes. This is impossible. So $X$ is uniruled.

REMARK 1.2. - A classical result in group actions on a projective manifold $X$ says that if $X$ carries a holomorphic vector field with zeroes, then $X$ is uniruled. If Question 1.6 had a positive answer, then we would be able to generalize this result to arbitrary tensor powers of the tangent bundle, and we may also allow a twist with a topologically trivial line bundle. In other words, we would be able to generalize (1.12) by assuming there only the existence of some zero without saying anything on the dimension of the zero locus.

Although the methods of this section basically fail in the Kähler case, it seems reasonable to make the following

ConjeCtuRe 1.11. - Let $X$ be an n-dimensional compact Kähler manifold,

$$
\left(\Omega_{X}^{1}\right)^{\otimes m} \rightarrow Q \rightarrow 0
$$

a torsion free quotient. If $X$ is not uniruled, then

$$
c_{1}(Q) \cdot \pi_{*}\left(\omega_{1} \cdots \omega_{n-1}\right) \geq 0
$$

for all bimeromorphic maps $\pi: X^{\prime} \rightarrow X$ from any compact Kähler manifold $X^{\prime}$ and all Kähler forms $\omega_{j}$ on $X^{\prime}$. 


\section{A characterization of varieties of general type}

2.1. Refined Kodaira Dimension. - The following "refined Kodaira dimension" was introduced in [3]. It measures the geometric positivity of the cotangent bundle, and not only that of the canonical bundle. Its definition will be justified in the next subsection.

DeFinition 2.1. - Let $X$ be a compact (or projective) manifold. Then $\kappa^{+}(X)$ is the maximal number $\kappa(\operatorname{det} \mathcal{F})$, where $\mathcal{F} \subset \Omega_{X}^{p}$ for $1 \leq p \leq \operatorname{dim} X$ is a (saturated) coherent subsheaf.

Obviously we have $\kappa^{+}(X) \geq \kappa(X)$ for any $X$.

Assuming the standard conjectures of the Minimal Model Program, one can easily describe $\kappa^{+}(X)$ as follows (see [3] for details, where the following conjecture was formulated):

Conjecture 2.2. - Let $X$ be a projective manifold. If $X$ is not uniruled (or if $\kappa(X) \geq 0)$, then $\kappa^{+}(X)=\kappa(X)$.

When $X$ is uniruled, one has

$$
\kappa^{+}(X)=\kappa^{+}(R(X)),
$$

where $R(X)$ is the so-called "rational quotient" of $X$; see [3]. This rational quotient is not uniruled, and so should be either one point or have $\kappa^{+}(R(X))=$ $\kappa(R(X)) \geq 0$. Thus if $X$ is uniruled, one has $\kappa(X)=-\infty$ but $\kappa^{+}(X) \geq 0$, unless $R(X)$ is one point, which means that $X$ is rationally connected. In this latter case $\kappa^{+}(X)=-\infty$. Conversely, if $\kappa^{+}(X)=-\infty$, then $X$ should be rationally connected.

Notice that $\chi\left(\theta_{X}\right)=1$ if $\kappa^{+}(X)=-\infty$, because $h^{0}\left(X, \Omega_{X}^{p}\right)=0$ for $p>0$. In [3] it is shown that $X$ is simply connected if $\kappa^{+}(X)=-\infty$ which of course is also true for $X$ rationally connected.

The above conjecture is a geometric version of the stability of the cotangent bundle of $X$ when $X$ is not uniruled. It is a version in which positivity of subsheaves is measured by the Kodaira dimension of the determinant bundle, and not by the slope after restricting to "strongly movable curves".

2.2. A Characterisation of Varieties of General Type. - As a consequence of the preceeding criteria for uniruledness, we first solve the above conjecture in the extremal case when $\kappa^{+}(X)=n$ (we shall study in the next section below the intermediate cases):

TheOREm 2.3. - Let $X$ be an n-dimensional projective manifold and suppose $\kappa^{+}(X)=n$, i.e., some $\Omega_{X}^{p}$ contains a subsheaf $\mathcal{F}$ with $\kappa(\operatorname{det} \mathcal{F})=n$. Then $\kappa(X)=n$. 
Proof. - First let us see that $X$ is not uniruled. In fact, otherwise take a covering family of rational curves and select a general member $C$ so that $T_{X} \mid C$ is nef. Hence the dual of $\Omega_{X}^{p} \mid C$ is nef and therefore $\mathcal{F} \mid C$ cannot have ample determinant. So $X$ cannot be uniruled.

Of course, we may assume that $\mathcal{F}$ saturated, hence $Q=\Omega_{X}^{p} / \mathcal{F}$ is torsion free. By taking determinants we get

$$
m K_{X}=\operatorname{det} \mathcal{F}+\operatorname{det} Q
$$

for some positive integer $m$. We learn from (1.6) above that $\operatorname{det} Q$ is pseudoeffective. Thus $K_{X}$ is big, as a sum of a big and a pseudo-effective divisor.

2.3. The intermediate case. - In this section we want to study the above Conjecture 2.2 in the intermediate case $n>\kappa\left(X_{n}\right) \geq 0$.

We shall reduce Conjecture 2.2 to (special cases of) a seemingly considerably simpler:

Conjecture 2.4. - Let $X$ be a projective manifold. Let $N K_{X}=A+B$ with some positive integer $N>0, A$ effective and $B$ pseudo-effective. Then $\kappa(X) \geq \kappa(A)$.

Remark 2.1. - (1) By suitably blowing up, it is easily seen that Conjecture 2.4 is equivalent to the analogous conjecture with $A$ spanned.

(2) If $\nu(L)$ denotes the numerical dimension of an arbitrary pseudo-effective line bundle as introduced by Boucksom [1], then the generalised abundance conjecture states

$$
\kappa\left(K_{X}\right)=\nu\left(K_{X}\right) .
$$

If this generalised abundance conjecture holds, then Conjecture 2.4 holds when $\kappa(X)=0$, a case sufficient to imply Conjecture 2.2 (see below). In fact, if $\kappa\left(K_{X}\right)=0$ and $N K_{X}=A+B$ with $A$ spanned and $B$ pseudo-effective, then $\nu(A+B)=0$, hence $\nu(A)=0$ and therefore $A=0, A$ being spanned.

We start with an immediate observation:

Proposition 2.5. - Conjecture 2.4 implies Conjecture 2.2, when $X$ is not uniruled (in particular when $\kappa(X) \geq 0$ ).

Proof. - Let $\mathcal{F}$ be a saturated subsheaf of $\Omega_{X}^{p}$ such that $\kappa(X, \operatorname{det}(\mathcal{F}))=$ $\kappa^{+}(X) \geq 0$, then $Q=\Omega_{X}^{p} / \mathcal{F}$ is torsion free. By taking determinants we get

$$
m K_{X}=\operatorname{det} \mathcal{F}+\operatorname{det} Q
$$

for some positive integer $m$. We know that $\operatorname{det} Q$ is pseudo-effective, because $X$ is not uniruled. By Conjecture 2.4, we get the claim, since $A:=\operatorname{det}(\mathscr{F})$ is $\mathbb{Q}$-effective. 
We now show that Conjecture 2.4 (in case $\kappa(X) \geq 0$ ) - and thus also 2.2 -is a consequence of the special case $\kappa(X)=0$ of Conjecture 2.4. More precisely:

Proposition 2.6. - Let $X$ be a projective $n$-dimensional manifold with $\kappa(X) \geq 0$. Let $d=n-\kappa(X) \geq 0$. If Conjecture 2.4 holds for all manifolds $G$ of dimension $d$ and with $\kappa(G)=0$, then Conjecture 2.4 (and thus also Conjecture 2.2) holds for $X$.

Proof. — By blowing up we may assume that the Iitaka fibration $g: X \rightarrow W$ is holomorphic. Let $G$ be a general fiber of $g$. Thus $\kappa(G)=0$. Let $A$ be effective and $B$ pseudo-effective on $X$ such that

$$
N K_{X}=A+B
$$

for some positive integer $N$. Then $A_{G}$ is effective, $B_{G}$ is pseudo-effective and

$$
N K_{G}=A_{G}+B_{G} .
$$

Thus by Conjecture 2.4 applied to $G$, we conclude that $\kappa\left(G, A_{\mid G} \leq 0\right.$. By the easy additivity theorem for the Kodaira dimension, we obtain that

$$
\kappa(X, A) \leq \operatorname{dim}(W)+\kappa\left(G, A_{\mid G}\right) \leq \operatorname{dim}(W)=\kappa(X) .
$$

The preceeding observation shows that the only two crucial cases of Conjecture 2.4 are $\kappa(X)=0$ and $\kappa(X)=-\infty$.

We now present some circumstances in which Conjecture 2.4 can be solved, so that 2.6 can be applied.

We first recall a notion from Mori theory. Let $X$ be a projective manifold. A variety $X^{\prime}$ with at most terminal singularities is said to be a good minimal model for $X$, if $X^{\prime}$ is birational to $X$ and some $m K_{X^{\prime}}$ is (locally free and) spanned. Good minimal models are predicted to exist for every $X$ with $\kappa(X) \geq 0$ but this known only in dimension up to 3 .

Proposition 2.7. - Let $G$ be a projective manifold with $\kappa(G)=0$. Suppose $G$ has a good minimal model and that

$$
N K_{G}=A+B
$$

with $A$ effective and $B$ pseudo-effective. Then $\kappa(A)=0$.

Proof. - Let $G^{\prime}$ be a good minimal model for $G$. Then $K_{G^{\prime}} \equiv 0$ and actually $K_{G^{\prime}}$ is torsion. Choose a smooth model $\hat{G}$ with holomorphic maps $\pi: \hat{G} \rightarrow G$ and $\lambda: \hat{G} \rightarrow G^{\prime}$. There is an effective divisor $E$ supported on the exceptional locus of $\pi$ such that $K_{\hat{G}}=\pi^{*}\left(K_{G}\right)+E$. Then we can write

$$
N K_{\hat{G}}=\hat{A}+\hat{B}
$$

TOME $139-2011-\mathrm{N}^{\mathrm{O}} 1$ 
with $\hat{A}=\pi^{*}(A)+N E$ effective and $\hat{B}=\pi^{*}(B)$ pseudo-effective. Now consider $A^{\prime}=\lambda_{*}(\hat{A})$ and $B^{\prime}=\lambda_{*}(\hat{B})$. Then $A^{\prime}$ is effective, $B^{\prime}$ is pseudo-effective and

$$
N K_{G^{\prime}}=A^{\prime}+B^{\prime} .
$$

It follows $A^{\prime}=B^{\prime}=0$ so that $\kappa(A)=0$.

Since good minimal models exist in dimension up to 3, Prop. 2.6 gives in particular:

Theorem 2.8. - Let $X$ be a projective $n$-dimensional manifold, $\kappa(X) \geq 0$. Suppose $\kappa(X) \geq n-3$. Then $\kappa^{+}(X)=\kappa(X)$.

For some other result towards (2.4) we state

Proposition 2.9. - Let $X$ be a projective $n$-dimensional manifold, $N K_{X}=$ $A+B$ with $A$ spanned and $B$ pseudo-effective. Let $f: X \rightarrow Y$ be the fibration determined by $|A|$. Let $F$ be the general fiber of $f$. If $B \mid F$ is big, then $K_{X}$ is big, i.e., $\kappa(X)=n$.

Proof. - This is proved in $[4,2.5]$.

Corollary 2.10. - Let $X_{n}$ be a projective manifold, $N K_{X}=A+B$ with $A$ spanned and $B$ pseudo-effective. If $\kappa(A)=n-1$, then $\kappa(X) \geq n-1$.

Proof. - Let $f: X \rightarrow Y$ be the fibration associated with $A$ and let $F$ denote the general fiber. Since $\operatorname{dim} F=1$, either $B_{F}$ is ample or $B_{F} \equiv 0$.

In the first case we simply apply (2.10). In the second we notice $N K_{F}=$ $B_{F} \equiv 0$ so that $F$ is elliptic and $B_{F}=0$. Then we can write

$$
m B=f^{*}(L)+\sum d_{i} D_{i}
$$

with $L$ a line bundle on $Y$, with $d_{i}$ integers, not necessarily positive, and with $D_{i}$ irreducible divisors with $\operatorname{dim} f\left(D_{i}\right) \leq n-3$. We want to show that $L$ is pseudo-effective.

Restricting to an irreducible curve $C \subset Y$ going through a fixed, but general point of $Y$, we see that $B_{\mid X_{C}}$ is still pseudo-effective, where $X_{C}$ is the main component of $f^{-1}(C)$. Blowing-up $X_{C}$ if necessary, we may assume that $C, X_{C}$ are smooth. We are then reduced to the case where $Y=C$ is a curve. But then there are no exceptional divisors $D_{i}$, and the degree of $L_{\mid C}$ is nonnegative, as desired.

Writing $A=f^{*}\left(A^{\prime}\right)$, it follows that $A^{\prime}+L$ is big since $A^{\prime}$ is big, and $L$ is pseudo-effective. Hence

$$
\kappa\left(N m K_{X}+\sum\left(-d_{i}^{\prime} D_{i}\right)\right)=n-1,
$$


where $d_{i}^{\prime}$ are just the negative $d_{i}$. Then however

$$
\kappa(X) \geq n-1
$$

too.

\section{Numerical maximality of the Kodaira dimension}

We solve here Conjecture 2.4 in the special case where $B$ is numerically trivial.

THEOREM 3.1. - Let $X$ be a projective complex manifold, and $L \in \operatorname{Pic}^{0}(X)$ be numerically trivial. Then:

1. $\kappa\left(X, m K_{X} \otimes L\right) \leq \kappa(X)$.

2. If $\kappa(X)=0$, and if $\kappa\left(X, m K_{X} \otimes L\right)=\kappa(X)$, then $L$ is a torsion element in the group $\operatorname{Pic}^{0}(X)$.

REMARK 3.1. - The conclusion of (2) above does no longer hold when $\kappa(X) \geq$ 1 , as shown by curves (or even arbitrary manifolds) of general type.

Another point not shown by our arguments is the behaviour of the modified plurigenera

$$
p_{m}^{+}(X):=\sup \left\{h^{0}\left(X, m K_{X} \otimes L\right), L \equiv 0\right\},
$$

as $m$ is large and divisible. One may expect that then $p_{m}^{+}(X)=p_{m}(X)$, and that the maximum is attained at a torsion point, for every $m>0$ (this is true for $m=1$, by the arguments below).

Proof. - We first reduce the general case where $\kappa(X)>0$ to the special case $\kappa(X)=0$, as in 2.6 above.

Observe first that the statements involved are preserved by birational transformations of $X$. We can thus assume that both $f, g$ are holomorphic, where $g: X \rightarrow W$ is the Iitaka-Moishezon fibration of $X$ defined by some $\left|m K_{X}\right|$, and $f: X \rightarrow Y$ is the Iitaka fibration defined by some $\left|m\left(K_{X} \otimes L\right)\right|$. If $G$ is a general fibre of $g$, then it is sufficient to show that $f(G)$ is a single point of $Y$. But then $f_{\mid G}$ is nothing but the Iitaka fibration on $G$ defined by $\left(K_{X} \otimes L\right)_{\mid G}$. Because $\kappa(G)=0$, we obtain the conclusion from the special case $\kappa=0$.

REMARK 3.2. - We see moreover that, in order to have equality $\kappa\left(K_{X} \otimes L\right)=$ $\kappa(X)$, it is necessary that $L_{\mid G}$ be torsion, by claim (2) for $\kappa=0$.

To conclude the proof of the preceeding theorem, we need to solve the case $\kappa(X) \leq 0$. This is the content of the next two propositions.

We first deal with the case $m=1$.

Proposition 3.2. - Let $X$ be a projective manifold, $L \in \operatorname{Pic}^{0}(X)$. If $h^{0}\left(K_{X} \otimes L\right) \geq r>0$, then the following holds. 
1. There exists a finite étale abelian cover $f: \tilde{X} \rightarrow X$ such that:

$$
h^{0}\left(f^{*}\left(K_{X} \otimes t \cdot L\right)\right)=h^{0}\left(K_{\tilde{X}} \otimes f^{*}(t \cdot L)\right) \geq r
$$

for all $t \in \mathbb{R}$.

(here $t \cdot L$ denotes any element $L_{t}$ in any one-parameter subgroup of $\operatorname{Pic}^{0}(X)$ containing $\left.L\right)$

2. $h^{0}\left(K_{\tilde{X}}\right) \geq r$.

3. In particular, $\kappa(X) \geq r-1 \geq 0$, if $r=1$ or $r=2$.

4. If $\kappa(X)=0$ and if $h^{0}\left(K_{X} \otimes L\right)=1$, then $L$ is a torsion element in the group $\operatorname{Pic}^{0}(X)$.

Proof. - Assuming (1) for the moment, we choose $t=0$ and obtain (2) and therefore also (3).

We next prove (1). Let

$$
S_{X}^{m}:=\left\{L \in P i c^{0}(X) \mid H^{m}(X, L) \neq 0\right\} .
$$

By Simpson [25]:

$$
S_{X}^{m}=\bigcup\left\{A_{i}+T_{i}\right\}
$$

with $A_{i}$ torsion elements and $T_{i}$ subtori of $\operatorname{Pic}^{0}(X)$.

Choose a finite abelian étale cover $f: \tilde{X} \rightarrow X$ such that $f^{*}\left(A_{i}\right)=\theta_{\tilde{X}}$ for all $i$. Applying Simpson's result with $m=\operatorname{dim} X$ and using Serre duality, we conclude

$$
h^{0}\left(K_{\tilde{X}} \otimes f^{*}(t \cdot L)\right) \geq r
$$

for all $t \in \mathbb{R}$.

Indeed:

$$
f^{*}(t \cdot L) \in f^{*}\left(T_{i}\right)=f^{*}\left(A_{i}+T_{i}\right)
$$

for some $i$ such that $L \in A_{i}+T_{i}$. Since $f^{*}\left(A_{i}+T_{i}\right) \subset f^{*}\left(S_{X}^{r}\right) \subset S_{\tilde{X}}^{r}$, we are done for assertion (1).

(Notice that if $L$ is unitary flat, then by Hodge theory it is obvious that $h^{0}\left(K_{X} \otimes L\right)=h^{0}\left(K_{X} \otimes L^{*}\right)$, without using [25]).

Let us finally prove statement (4). We show by contradiction that $T_{i}$ is the trivial group. Replacing $X$ by $\tilde{X}$ as above and setting $r=1$, we get a non-trivial one-parameter subgroup $L_{t}, t \in \mathbb{R}$, contained in $T_{i} \subset S_{X}^{n}$, where $n=\operatorname{dim} X$. The canonical morphisms

$$
H^{0}\left(K_{\tilde{X}} \otimes L_{t}\right) \otimes H^{0}\left(K_{\tilde{X}} \otimes L_{t}^{*}\right) \rightarrow H^{0}\left(2 K_{\tilde{X}}\right)
$$

show that $h^{0}\left(2 K_{\tilde{X}}\right) \geq 2$, contradicting our assumption that $\kappa(X)=0$.

We shall now reduce the general case of $m \geq 2$ to the special case $m=1$, by means of cyclic covers. 
THEOREM 3.3. - Let $X$ be a projective manifold and $L$ a line bundle with $c_{1}(L)=0$ in $H^{2}(X, \mathbb{Z})$.

1. Suppose that there is a positive integer $m$ such that $h^{0}\left(m K_{X} \otimes L\right) \geq 2$. Then $\kappa(X) \geq 1$.

2. Suppose that there is a positive integer $m$ such that $h^{0}\left(m K_{X} \otimes L\right) \neq 0$. Then $\kappa(X) \geq 0$.

3. Suppose that $\kappa(X)=0$, and that $h^{0}\left(m K_{X} \otimes L\right) \neq 0$. Then $L$ is torsion in $\operatorname{Pic}^{0}(\mathrm{X})$.

Proof. - We first prove (1), the proof of (2) being identical, simply omitting the divisor $D$ in the arguments below. Since our claim is invariant by finite étale covers, we can pass to such covers as we like. In particular, we may assume that $L \in \operatorname{Pic}^{0}(X)$. If $m=1$, then our claim is Proposition 3.4, hence we shall assume $m \geq 2$. Furthermore we may assume that $L=m L^{\prime}$, so that

$$
h^{0}\left(m\left(K_{X} \otimes L^{\prime}\right)\right) \geq 2 .
$$

Let $\sum b_{i} B_{i}$ be the fixed part of $\left|m\left(K_{X} \otimes L^{\prime}\right)\right|$, so that we can write

$$
m\left(K_{X} \otimes L^{\prime}\right)=\sum b_{i} B_{i}+D
$$

with $D$ reduced and movable. By possibly blowing up we may assume that the support of $\sum b_{i} B_{i}+D$ has normal crossings. Now take the $m$-th root, normalize and desingularize to obtain $f: Y \rightarrow X$. We have to compute $f_{*}\left(K_{Y}\right)$, following $[8,26]$. In fact, introduce the line bundles

$$
H_{j}=j\left(K_{X} \otimes L^{\prime}\right)-\sum\left[j b_{i} m^{-1}\right] B_{i} .
$$

Here $[\mathrm{x}]$ denotes the integral part of $x$. Then:

$$
f_{*}\left(K_{Y}\right)=K_{X} \otimes \bigoplus_{j=0}^{m-1} H_{j}
$$

Hence the direct summand of $f_{*}\left(K_{Y}\right) \otimes L^{\prime}$ corresponding to $j=m-1$ is just

$$
D+\sum_{i}\left(b_{i}-\left[b_{i}(m-1) m^{-1}\right]\right) B_{i}
$$

Since $D$ moves, we obtain

$$
h^{0}\left(f_{*}\left(K_{Y}\right) \otimes L^{\prime}\right) \geq 2,
$$

hence

$$
h^{0}\left(K_{Y} \otimes f^{*}\left(L^{\prime}\right)\right) \geq 2
$$

so that $\kappa(Y) \geq 1$.

We still need to prove $\kappa(X) \geq 1$. As already indicated above, the map $f: Y \rightarrow X$ decomposes as

$$
f=h_{2} \circ h_{1} \circ h_{0},
$$

TOME $139-2011-\mathrm{N}^{\mathrm{O}} 1$ 
in the following way: we first take the cyclic covering $h_{0}: Y_{0} \rightarrow X$ determined by $m\left(K_{X} \otimes L^{\prime}\right)=\vartheta_{X}(D)$. Then we take the normalisation $h_{1}: Y_{1} \rightarrow Y_{0}$, and finally take $h_{2}: Y \rightarrow Y_{1}$ to be a desingularisation. Then $Y_{0}$ is Gorenstein and

$$
K_{Y_{0}}=h_{0}^{*}\left(m K_{X} \otimes(m-1) L^{\prime}\right) ;
$$

furthermore

$$
K_{Y_{1}} \subset h^{*}\left(K_{Y_{0}}\right)
$$

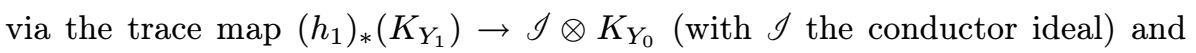
finally

$$
\left(h_{2}\right)_{*}\left(K_{Y}\right)=K_{Y_{1}}
$$

since $Y_{1}$ has rational singularities $[8,26]$.

In total

$$
\left.K_{Y} \subset f^{*}\left(m K_{X} \otimes(m-1) L^{\prime}\right)\right) \otimes \vartheta_{Y}\left(\sum a_{i} E_{i}\right)
$$

where $E_{i}$ are the exceptional components for $h_{2}$, and the $a_{i}^{\prime} s$ are integers.

Hence:

by $(+)$. Thus

$$
\left(h_{2}\right)_{*}\left(\theta_{Y}\left(\sum a_{i} E_{i}\right)\right)=\theta_{Y_{1}}
$$

$$
K_{Y} \otimes f^{*}\left((1-m) L^{\prime}\right) \subset f^{*}\left(m K_{X}\right) \otimes \vartheta_{Y}\left(\sum a_{i} E_{i}\right) .
$$

From the assertion (2) of Proposition 3.2, we conclude that, for some abelian étale cover $g: \tilde{Y} \rightarrow Y$, we have $h^{0}\left(K_{\tilde{Y}}\right) \geq 2$. Thus $\kappa(X)=\kappa(\tilde{Y}) \geq 1$, as claimed.

The proof of (3) is then the same as the proof of (4) in 3.2.

REMARK 3.3. - The preceeding result makes plausible the expectation that the generalised Green-Lazarsfeld sets

$$
S_{m, p, r}=\left\{L \in \operatorname{Pic}^{0}(X) \mid h^{p}\left(m K_{X} \otimes L\right) \geq r\right\}
$$

might have the same structure as in [25] (finite union of translates of subtori by torsion elements).

In fact, up to the word "torsion" above, this is a consequence of the Abundance Conjecture, as C. Mourougane observed. Indeed he showed in [21], thm. 5.3 , that the Green-Lazarsfeld cohomological loci have this structure for "good" divisors.

Corollary 3.4. - Let $X$ be a projective manifold, $A$ effective and $B$ pseudoeffective divisors on $X$. Assume that $m K_{X}=A+B$ for some positive integer $m$. Suppose also that $\nu(B)=0$, in the sense of [1]. Then $\kappa(X) \geq 0$.

Proof. - By [1], we can write $B \equiv \sum b_{i} B_{i}$ with positive rational numbers $b_{i}$. Now apply (3.1). 


\section{The Universal Cover}

Another invariant of $X$ is defined via the universal cover $\tilde{X}$ of a compact Kähler or projective manifold $X$. By identifying points in $\tilde{X}$ which can be joined by a compact connected analytic set, one obtains an almost holomorphic meromorphic map $\tilde{X} \rightarrow \Gamma(\tilde{X})$. Here "almost holomorphic" is to say that the degeneracy locus does not project onto the image. If $\tilde{X}$ is holomorphically convex (which is expected to be always true by the so-called Shafarevitch conjecture), then this map is holomorphic and is just the usual Remmert holomorphic reduction. In any case it induces the so-called Shafarevich map

$$
\gamma_{X}: X \rightarrow \Gamma(X)=\Gamma(\tilde{X}) / \pi_{1}(\tilde{X}) .
$$

Definition 4.1. $-\gamma d(X)=\operatorname{dim} \Gamma(X)$ is the $\Gamma$-dimension of $X$.

Notice that $\gamma d(X)=0$ iff $\pi_{1}(X)$ is finite and that $\gamma d(X)=\operatorname{dim} X$ iff through the general point of $\tilde{X}$ there is no positive dimensional compact subvariety, i.e., $\tilde{X}$ geometrically seems as a modification of a Stein space.

The following result $[3,(4.1)]$ gives a relation between $\kappa^{+}(X)$ and $\gamma d(X)$.

THEOREM 4.2. - Let $X$ be a compact Kähler manifold. If $\chi\left(X, \theta_{X}\right) \neq 0$, then either

1. $\kappa^{+}(X) \geq \gamma d(X)$, or

2. $\kappa^{+}(X)=-\infty$, and so $X$ is simply connected.

By (2.9) we then obtain

Corollary 4.3. - Let $X_{n}$ be a projective manifold. Suppose that $\kappa(X) \geq$ $n-3$ and $\chi\left(\theta_{X}\right) \neq 0$. Then $\kappa(X)=\kappa^{+}(X) \geq \gamma d(X)$.

In particular, if $n=4, \kappa(X) \geq 1, \pi_{1}(X)$ is infinite and $\chi\left(\theta_{X}\right) \neq 0$, then $\kappa(X) \geq \gamma d(X) \geq 1$. In other words, if $X$ is a projective 4-fold with $\kappa(X)=0$ and $\pi_{1}(X)$ is not finite, then either $\chi\left(\theta_{X}\right)=0$; so there is either a holomorphic 1-form, or a holomorphic 3-form, or: $\kappa^{+}(X) \in\{1,2,3\}$.

Hence as in $[3,5.9]$, we conclude:

Corollary 4.4. - Let $X$ be a projective manifold of dimension 4 such that $\kappa(X)=0$, and $\chi\left(\theta_{X}\right) \neq 0$. Then either $\pi_{1}(X)$ is finite and has at most 8 elements, or $\kappa^{+}(X) \in\{1,2,3\}$.

This result should hold in arbitrary dimension $n$, with 8 replaced by $2^{n-1}$, as a consequence of the standard conjecture that $\pi_{1}(X)$ should be almost abelian if $\kappa(X)=0$.

From Theorem 2.3 we deduce

TOME $139-2011-\mathrm{N}^{\mathrm{O}} 1$ 
Corollary 4.5. - Let $X$ be a normal projective variety with at most rational singularities and suppose that its universal cover is not covered by its positivedimensional compact subvarieties. Then $X$ is of general type if $\chi\left(\theta_{X}\right) \neq 0$.

Proof. - If $X$ is smooth, then by our assumption and (4.2), we have $\kappa^{+}(X)=$ $\operatorname{dim} X$ or $\chi\left(\theta_{X}\right)=0$. Now theorem (2.3) gives the claim.

So it remains to reduce the general case to the smooth. Note that $\tilde{X}$ is irreducible since $X$ is normal. Consider a projective desingularisation $\pi: Y \rightarrow$ $X$ and let $\tilde{\pi}: \tilde{Y} \rightarrow \tilde{X}$ be the induced maps on the level of universal covers. Then $\tilde{\pi}$ is onto with discrete fibers over the smooth locus of $\tilde{X}$. Hence $\tilde{Y}$ is not covered by positive-dimensional compact subvarieties, too, because their $\tilde{\pi}$-images would again be compact. By the solution of the smooth case, we either have $\chi\left(X, \theta_{Y}\right)=0$--hence $\chi\left(\theta_{X}\right)=0$ by the rationality of the singularities of $X$--or $Y$, hence $X$, is of general type.

Corollary 4.6. - Let $X_{n}$ be a projective manifold or a normal projective variety with at most terminal singularities whose universal cover is Stein (or has no positive-dimensional subvariety). Then either $K_{X}$ is ample or $\chi\left(\theta_{X}\right)=$ $0, K_{X}$ is nef and $K_{X}^{n}=0$.

Proof. - This is immediate from (4.5) by observing that $X$ does not have any rational curve, so that $K_{X}$ must be nef by Mori theory. Moreover if $K_{X}$ is big, then $K_{X}$ is ample by Kawamata [12].

We are lead to ask for the structure of projective manifolds $X_{n}$ whose universal cover is Stein and with $K_{X}^{n}=0$.

ConjeCture 4.7. - Let $X_{n}$ be a projective manifold whose universal cover $\tilde{X}$ is Stein. Assume $K_{X}^{n}=0$. Then up to finite étale cover of $X$, the manifold $X$ has a torus submersion over a projective manifold $Y$ with $K_{Y}$ ample and universal cover again Stein.

If the universal cover is only assumed not to admit a positive-dimensional subvariety through the general point, then one expects a birational version of 4.7 , which is actually proved in $[13,5.8]$. Here is the "Stein version" of this result which does not follow immediately from Kollár's result since we make a biholomorphic statement. The main point is to explain that we must have a holomorphic Iitaka fibration which is "almost smooth" and then apply Kollár's techniques to make it smooth.

Proposition 4.8. - Conjecture 4.7 holds if $\kappa(X) \geq n-3$. 
Proof. - (1) Since the case $\kappa(X)=n-1$ is the simplest, we do it first. Here the numerical dimension $\nu(X)=\kappa(X)$, so that $K_{X}$ is good, i.e., some multiple is spanned [11]. Therefore we have a holomorphic Iitaka fibration $f: X \rightarrow Y$. The general fiber is an elliptic curve. Since $X$ does not contain rational curves, it follows easily that all fibers are elliptic, sometimes multiple. Now [13, sect.6] yields a finite étale cover such that the induced map is smooth; see below for some details.

(2) In the other case we consider the normalized graph $p: \mathscr{C} \rightarrow X$ of the family determined by the general fibers of the meromorphic Iitaka fibration. Let $q: \mathscr{C} \rightarrow T$ denote the parameter space. All irreducible fibers of $q$ have dimension 2 (resp. 3) and every such fiber is an étale quotient of a torus by Lemma 4.9 below. Now we have a formula (via the trace map)

$$
K_{\mathscr{C}}=p^{*}\left(K_{X}\right)+E
$$

with an effective (Weil) divisor $E$. Restricting to a general (normal, hence smooth by (4.8) below) fiber $F$ of $q$, we get

$$
0 \equiv p^{*}\left(K_{X}\right)|F+E| F \text {. }
$$

Hence $p^{*}\left(K_{X}\right)|F \equiv 0=E| F$. Now consider the reduction $F_{0}$ of a component of a singular fiber (or rather its normalization) and use the conservation law (and the nefness of $p^{*}\left(K_{X}\right)$ ) to deduce $p^{*}\left(K_{X}\right) \mid F_{0} \equiv 0$. Thus $p^{*}\left(K_{X}\right)$ is " $q$-numerically trivial". This proves immediately $\nu(X)=n-2($ resp. $\nu(X)=n-3)$ and again $m K_{X}$ is spanned for a suitable $m$.

Now let again $F_{0}$ be the reduction of a component of a singular fiber $F$, this time of the Iitaka fibration $f: X \rightarrow Y$.

We claim that actually $F=a F_{0}$ for some integer $a>0$, and that $f$ is equidimensional.

If $\operatorname{dim} T=2$, this is easy and well-known of course (take a general curve through $f\left(F_{0}\right)$ and observe that singular non-multiple fibers produce rational curves).

So suppose $\operatorname{dim} T=1$. Take $\mu$ maximal such that $\mu F_{0} \subset F$. Then $N_{F_{0}}^{* \mu}$ has a section which has a zero, since $F$ is reducible. Hence $K_{F_{0}}=-D$ with $D$ a $\mathbb{Q}$-effective divisor by the adjunction formula. Now normalize and then desingularize. The result $\hat{F}_{0}$ has $\left.\kappa\left(\hat{F}_{0}\right)\right)=-\infty$ (use formula (*) below), so that $F_{0}$ is uniruled. Since this is forbidden by the universal cover, we obtain $F=a F_{0}$.

Then $K_{F_{0}} \equiv 0$, so that its normalization $\tilde{F}_{0}$ has

$$
K_{\tilde{F}_{0}}=\nu^{*}\left(K_{F_{0}}\right)-\tilde{N}
$$

with $\tilde{N}$ the preimage of the non-normal locus. Since $K_{\tilde{F}_{0}} \equiv 0$ by $(2.9)$, we conclude that $F_{0}$ must have been normal, hence smooth. 
Now we apply $[13,5.8]$ to obtain a finite étale cover $X^{\prime}$ of $X$ which is birational to a torus submersion. But since $X^{\prime}$ does not contain rational curves, we obtain a holomorphic birational map from a torus submersion to $X^{\prime}$. Since multiple fibers cannot be resolved by birational transformations on the base, we conclude that $X^{\prime}$ is a torus submersion itself.

It remains to prove the following lemma of independent interest.

LEMMA 4.9. - Let $X$ be an irreducible reduced variety of dimension at most 3. Assume that the universal cover of $X$ is Stein (or does not contain compact subvarieties). Let $\tilde{X} \rightarrow X$ be the normalization and $\pi: \hat{X} \rightarrow \tilde{X}$ be a desingularization. Suppose $\kappa(\hat{X})=0$. Then $\tilde{X}$ is an étale quotient of a torus.

Proof. - We only treat the case $\operatorname{dim} X=3$, the surface case being easier and left to the reader. By [22], $\hat{X}$ admits a finite étale cover $h: X^{\prime} \rightarrow \hat{X}$ which is birational to a product of a simply connected manifold and an abelian variety. By our assumption on the universal cover, the simply connected part does not appear. It follows that the Albanese map $\alpha: X^{\prime} \rightarrow A$ is birational. Now all irreducible components of all non-trivial fibers $\alpha$ are filled up by rational curves ( $\alpha$ factors via Mori contractions). Since $\tilde{X}$ does not contain rational curves, the map $X^{\prime} \rightarrow \hat{X} \rightarrow \tilde{X}$ therefore factors over $\alpha$, i.e., we obtain a finite $\operatorname{map} g: A \rightarrow \tilde{X}$.

This map is étale in codimension 1 . In fact otherwise by the ramification formula $K_{A}=g^{*}\left(K_{\tilde{X}}\right)+R$ (as Weil divisors). Thus $-K_{\tilde{X}}$ is non-zero effective and therefore $\kappa(\hat{X})=-\infty$, contradiction.

We want to see that $\tilde{X}$ is actually smooth and an étale quotient of $A$. First notice that $\tilde{X}$ is $\mathbb{Q}$-Gorenstein (if $g$ has degree $d$, then $d K_{\tilde{X}}=\emptyset$ on the regular part of $\tilde{X}$, hence everywhere). Now we can compare the formulas

$$
K_{X^{\prime}}=h^{*} \pi^{*}\left(K_{\tilde{X}}\right)+\sum a_{i} E_{i}
$$

and

$$
K_{X^{\prime}}=\sum b_{j} F_{j}
$$

where $E_{i}$ are the preimages of the $\pi$-exceptional components and $F_{j}$ are the $\alpha$-exceptional components; notice $b_{j}>0$. Then both sets of exceptional divisors are equal, and thus all $a_{i}>0$. Therefore $\tilde{X}$ has only terminal singularities. We also notice that $\pi_{1}(\tilde{X})$ is almost abelian, i.e., abelian up to finite index. Therefore $\pi_{1}(\tilde{X})$ is abelian after finite étale cover. Then [10] applies and $\tilde{X}$ is an étale quotient of an abelian threefold. Here of course we use again that the universal cover of $\tilde{X}$ is Stein. 


\section{Stability and tensor products}

Recall that $\overline{M E}(X)$ denotes the movable cone of the $n$-dimensional projective manifold $X$. We say that $\alpha \in \overline{M E}(X)$ is geometric, if there exists a modification $\pi: \tilde{X} \rightarrow X$ from the projective manifold $\tilde{X}$ and ample line bundles $H_{i}$ such that

$$
\alpha=\lambda \pi_{*}\left(H_{1} \cap \cdots \cap H_{n-1}\right)
$$

with a positive multiple $\lambda$. By definition, $\overline{M E}(X)$ is the closed cone generated by the geometric classes.

A class $\alpha \in \overline{M E}(X) \cap H^{2}(X, \mathbb{Q})$ which is in the interior of $\overline{M E}(X)$ is called an rational ample class. Notice that a rational ample class is a linear combination of geometric classes.

If $\mathcal{E}$ and $\mathcal{F}$ are torsion free sheaves, then we put

$$
\mathcal{E} \hat{\otimes} \mathcal{F}=(\mathscr{E} \otimes \mathcal{F}) / \text { tor. }
$$

The first main result is well-known in case of an ample polarization $\left(H_{1}, \ldots, H_{n-1}\right.$.

THEOREM 5.1. - Let $\alpha \in \overline{M E}(X)$ be a rational ample class and let $\mathcal{E}$ and $\mathcal{F}$ be $\alpha$-semi-stable torsion free sheaves on $X$. Then $\mathcal{E} \hat{\otimes} \mathcal{F}$ is again $\alpha$-semi-stable.

The key to Theorem 5.1 is the following

Proposition 5.2. - Assume in the setup of (5.1) that $\mathcal{E}$ and $\mathcal{F}$ are locally free and $\alpha$-stable, where $\alpha \in \overline{M E}(X)$ be a rational ample class. Then $\mathcal{E} \otimes \mathcal{F}$ is $\alpha$-semi-stable.

Proof. - An analytic proof is given below in $\S 6$ by M. Toma.

An algebraic proof in case $\alpha$ is geometric is as follows, even for $\mathcal{E}$ and $\mathcal{F}$ only semi-stable. Since $\alpha$ is geometric, there is a modification $\pi: \tilde{X} \rightarrow X$ and ample line bundles $H_{i}$ on $\tilde{X}$ such that

$$
\lambda \alpha=\pi_{*}\left(H_{1} \cap \ldots H_{n-1}\right)=: \pi_{*}(h),
$$

we may of course assume $\lambda=1$ and all $H_{i}$ very ample. Since $E$ is $\alpha$-stable, so does $\pi^{*}\left(\mathcal{E}\right.$ w.r.t. $\pi^{*}(\alpha)$. By the projection formula the slope of $\pi^{*}(\mathcal{E})$ w.r.t. $\pi^{*}(\alpha)$ agrees with the $h$-slope (see Lemma 5.3), so that $\pi^{*}(\mathcal{E})$ is also $h$-stable. The same applies to $\pi^{*}(\mathcal{F})$. Now a well-known result (see e.g. [9, 3.1.4] says that $\pi^{*}(\mathscr{E} \otimes \mathcal{F})$ is $h$-stable, and therefore $\pi^{*}(\alpha)$-stable. Hence we conclude by Lemma 5.3 again. 
Let $\sigma: \hat{X} \rightarrow X$ be a modification from the projective manifold $\hat{X}$. Let $\alpha \in \overline{M E}(X)$ and $\hat{\alpha}=\sigma^{*}(\alpha)$ be the unique 1-cycle up to numerical equivalence in $\hat{X}$ such that

$$
\sigma^{*}(\alpha) \cdot \hat{L}=\alpha \cdot \sigma_{*}(\hat{L})
$$

for any line bundle $\hat{L}$ on $\hat{X}$. Here $\sigma_{*}(\hat{L})$ denotes the class of the line bundle $\sigma_{*}(\hat{L})^{* *}$. Alternatively, $\alpha$ defines a class in $H^{2 n-2}(X, \mathbb{R})$, where $n=\operatorname{dim} X$. Then $\sigma^{*}(\alpha)$ is the pull-back class in $H^{2 n-2}(\hat{X}, \mathbb{R})$.

We have in particular

$$
\sigma^{*}\left(\sigma_{*}(\beta)\right) \cdot \hat{L}=\sigma_{*}(\beta) \cdot\left(\sigma_{*}\left(\sigma^{*}(L)\right)=\sigma_{*}(\beta) . L\right.
$$

for any line bundle $L$ on $X$ and 1-cycle $\beta$ on $\hat{X}$. Our main interest is in applying this to the geometric class $\alpha=\sigma_{*}\left(H^{n-1}\right)$ as follows.

In order to deduce (5.1) from (5.2), we use the following lemma.

Lemma 5.3. - Let $\sigma: \hat{X} \rightarrow X$ be a modification from the projective manifold $\hat{X}$. Let $\alpha \in \overline{M E}(X)$ and $\hat{\alpha}=\sigma^{*}(\alpha)$. Then

1. $\hat{\alpha} \in \overline{M E}(\hat{X})$

2. If $\phi$ is a torsion free sheaf on $X$ and $\hat{S}=\sigma^{*}(\phi) /$ tor, then $\mu_{\alpha}(\phi)=\mu_{\hat{\alpha}}(\hat{\phi})$.

3. If $\hat{\phi}$ is torsion free on $\hat{X}$ and $\phi=\sigma_{*}(\hat{\phi})$, then $\mu_{\alpha}(\phi)=\mu_{\hat{\alpha}}(\hat{\phi})$.

4. A torsion free sheaf $\mathcal{E}$ on $X$ is $\alpha$-semi-stable if and only if $\sigma^{*}(\mathscr{E}) /$ tor is $\hat{\alpha}$-semi-stable.

Proof. - (1) We need to prove that $\hat{D} \cdot \hat{\alpha} \geq 0$ for all pseudo-effective divisors $\hat{D}$ on $\hat{X}$. Now the divisor $D=\sigma_{*}(\hat{D})$ is again pseudo-effective, see Lemma 5.4. Hence

$$
\hat{D} \cdot \hat{\alpha} \geq 0=D \cdot \alpha \geq 0
$$

proving (1).

(2) and (3) are simple calculations and (4) follows from (2) and (3).

More precisely, (2) is proved by

$$
\mu_{\hat{\alpha}}(\hat{\phi})=\hat{\alpha} \cdot c_{1}(\hat{\phi})=\hat{\alpha} \cdot \sigma^{*}\left(c_{1}(\varnothing)\right)=\alpha \cdot \sigma_{*}\left(\sigma^{*}\left(c_{1}(\phi)\right)=\alpha \cdot c_{1}(\phi)=\mu_{\alpha}(\varnothing),\right.
$$

whereas (3) is established by

$$
\mu_{\hat{\alpha}}(\hat{\phi})=\hat{\alpha} \cdot c_{1}(\hat{\phi})=\alpha \cdot \sigma_{*}\left(c_{1}(\hat{\phi})\right)=\alpha \cdot c_{1}\left(\sigma_{*}(\hat{\phi})\right)=\alpha \cdot c_{1}(\phi)=\mu_{\alpha}(\phi) .
$$

Lemma 5.4. - Let $\sigma: \hat{X} \rightarrow X$ be a birational morphism of compact Kähler manifolds. Let $\hat{L}$ be a pseudo-effective line bundle on $\hat{X}$. Then $L=\left(\pi_{*}(\hat{L})\right)^{* *}$ is pseudo-effective, too.

Proof. - The proof is very easy: since $\hat{L}$ is pseudo-effective, there exists a positive closed current $\hat{T}$ on $\hat{X}$ such that $c_{1}(\hat{L})=[\hat{T}]$. Now $T=\pi_{*}(\hat{T})$ is trivially again a positive closed current and $c_{1}(L)=[T]$. Therefore $L$ is pseudoeffective. 
Proof of Theorem 5.1. - We proceed by induction on $\operatorname{rk}(\mathcal{E})+\operatorname{rk}(\mathcal{F})$. We choose a birational morphism

$$
\sigma: \hat{X} \rightarrow X
$$

from a projective manifold $\hat{S}$ such that both $\hat{\mathcal{E}}=\sigma^{*}(\mathcal{E}) /$ tor and $\hat{\mathcal{F}}=\sigma^{*}(\mathcal{F}) /$ tor are locally free. We may moreover assume that $\sigma^{*}(\mathscr{E} \otimes \mathcal{F}) /$ tor is locally free, which implies

$$
\hat{\mathscr{E}} \otimes \hat{\mathcal{F}}=\sigma^{*}(\mathcal{E} \otimes \mathcal{F}) / \text { tor. }
$$

We consider

$$
\hat{\alpha}=\sigma^{*}(\alpha) \in \overline{M E}(\hat{X}),
$$

which is of course no longer an ample class (if $\sigma$ is not an isomorphism), i.e., it is not in the interior of $\overline{M E}(X)$. In order to be able to apply (5.2), we choose a sequence $\left(\alpha_{k}\right)$ of ample classes converging to $\alpha$ (in $N^{1}(X)$ or in $H^{2}(X)$ ); we can arrange $\alpha_{t}=\alpha+t\left(\omega_{1} \wedge \cdots \wedge \omega_{n-1}\right)$ with Kähler classes $\omega_{i}$.

Suppose first that $\mathcal{E}$ and $\mathcal{F}$ are $\alpha$-stable, not just semi-stable. Hence $\hat{\mathcal{E}}$ and $\hat{\mathcal{F}}$ are $\hat{\alpha}$-stable. By Lemma 5.6 the bundles $\hat{\mathcal{E}}$ and $\hat{\mathcal{F}}$ are $\alpha_{k}$-semi-stable for sufficiently large $k$. Then by $(5.2) \hat{\mathcal{E}} \otimes \hat{\mathcal{F}}$ is $\alpha_{k}$-semi-stable for large $k$. Thus $\hat{\mathcal{E}} \otimes \hat{\mathcal{F}}$ is $\hat{\alpha}$-semi-stable and we conclude again by Lemma 5.3 and (*).

If $\mathcal{E}$ and $\mathcal{F}$ are $\alpha$-semi-stable but not both stable, we proceed by induction on $\operatorname{rk}(\mathscr{E})+\operatorname{rk}(\mathcal{F})$. In case $\mathcal{E}$ is not stable, consider a stable subsheaf $\& \subset \mathcal{E}$ of strictly smaller rank and same slope as $\mathcal{E}$ and let $\mathcal{T}=\mathcal{F}$ (resp. in case $\mathcal{F}$ is not stable, let $\mathcal{T}$ be a stable subsheaf with the same slope and $\delta=\mathscr{E}$ ). By induction hypothesis $\phi \otimes \mathcal{T}$ and $\phi^{\prime} \otimes \mathcal{T}^{\prime}$ are semi-stable. Since

$$
\mu_{\alpha}(\varnothing)=\mu_{\alpha}\left(\phi^{\prime}\right)=\mu_{\alpha}(\mathcal{E})
$$

and

$$
\mu_{\alpha}(\mathcal{T})=\mu_{\alpha}\left(\mathcal{T}^{\prime}\right)=\mu_{\alpha}(\mathcal{F})
$$

it follows easily that $\mathcal{E} \otimes \mathcal{F}$ is $\alpha$-semi-stable. Namely, tensor the exact sequence

$$
0 \rightarrow \mathcal{T} \rightarrow \mathcal{F} \rightarrow \mathcal{T}^{\prime} \rightarrow 0
$$

by $\phi$ and $\phi^{\prime}$ to deduce the semi-stability of $\phi \hat{\otimes} \mathcal{F}$ and $\phi^{\prime} \hat{\otimes} \mathcal{F}$ and then tensor the the exact sequence

$$
0 \rightarrow \varnothing \rightarrow \mathcal{E} \rightarrow \phi^{\prime} \rightarrow 0
$$

by $\mathcal{F}$ to deduce the semi-stability of $\mathcal{E} \hat{\otimes} \mathcal{F}$. Here of course we need (1) and (2).

Corollary 5.5. - Let $\alpha \in \overline{M E}(X)$ be a rational ample class and $\mathcal{E}$ and $\mathcal{F}$ torsion free sheaves on $X$. Then $\mu_{\alpha}^{\max }(\mathcal{E} \hat{\otimes} \mathcal{F})=\mu_{\alpha}^{\max }(\mathcal{E})+\mu_{\alpha}^{\max }(\mathcal{F})$. 
Proof. - Let $\& \subset \mathcal{E}$ and $\mathcal{T} \subset \mathcal{F}$ be the maximal destabilizing sheaves. Since $\varnothing \hat{\otimes} \mathcal{T}$ is $\alpha$-semi-stable by Theorem 5.1 , we obtain

$$
\mu_{\alpha}^{\max }(\mathcal{E} \hat{\otimes} \mathcal{F}) \geq \mu_{\alpha}(\varnothing \hat{\otimes} \mathcal{T}) .
$$

Since $\mu_{\alpha}^{\max }(\mathcal{E})=\mu_{\alpha}(\delta)$, and analogously for $\mathcal{F}$ and $\mathcal{T}$, we conclude for one inequality. To establish the other, we must show that $\phi \hat{\otimes} \mathcal{T}$ is maximal destablisizing for $\mathcal{E} \hat{\otimes} \mathcal{F}$. This is an easy exercise using the exact sequences already used in the proof of (5.1) and the HN-filtration.

To deduce (5.1) from (5.2), we used the following:

LEMMA 5.6. - Let $X$ be a projective manifold of dimension $n$ and $\mathcal{E}$ a reflexive sheaf over $X$. Let $\alpha \in \overline{M E}(X) \cap H^{2 n-2}(X, \mathbb{Q})$ be a rational movable class. Choose rational Kähler classes $\omega_{i}$ and set

$$
\alpha_{t}=\alpha+t\left(\omega_{1} \wedge \cdots \wedge \omega_{n-1}\right)
$$

for $t \in \mathbb{R}_{+}$. Assume that $\mathcal{E}$ is $\alpha$-stable. Then $\mathcal{E}$ is $\alpha_{t}$-semi-stable if $|t|$ is sufficiently small.

Proof. - We assume to the contrary that $\mathcal{E}$ is $\alpha_{t_{j}}$-unstable for a sequence $t_{j}$ converging to 0 . Let $\phi_{t}$ denote the maximal destabilizing subsheaf with respect to $\alpha_{t}, t=t_{j}$. Let $r$ be the rank (assumed to be constant, as we can) of $\phi_{t}$. We shall use the shorthand

$$
\beta=\omega_{1} \wedge \cdots \wedge \omega_{n-1}
$$

and denote the slope w.r.t a classe $\gamma$ by $\mu_{\gamma}$, with the additional convention $\mu_{t}=\mu_{\alpha_{t}}$.

(1) We first show that

$$
\lim _{t \rightarrow 0} t \mu_{\beta}\left(\phi_{t}\right)=0 .
$$

In fact by taking $\bigwedge^{r_{t}}$ we are reduced to the following stronger statement.

Let $\mathcal{F}$ be a torsion free sheaf on a projective manifold. Let $\omega_{j}$ be rational Kähler classes. Then there is a positive constant $M$ such that for all locally free subsheaves $\mathcal{L} \subset \mathcal{F}$ of rank 1 the following inequality holds.

$$
c_{1}(\mathscr{L}) \cdot \beta \leq M
$$

This claim is clear: first reduce to the case that $\mathcal{F}$ is locally free by choosing a complete intersection curve $C$ of very ample divisors of large degree which are cohomologically multiples of the given Kähler classes. This curve will then avoid the non-free locus of $\mathcal{E}$. The claim is then obvious (by considering a filtration of $\mathcal{E}$ on $C$ by locally free sheaves of increasing ranks).

Since $\mu_{\beta}\left(\phi_{t_{j}}\right)$ is also bounded from below by $\mu_{\beta}(\mathcal{E})$ (use $(* *)$ ), the claim $(*)$ is proved. 
(2) Using $(*)$ we proceed as follows. The destabilizing property reads

$$
\left.\mu_{t}\left(\phi_{t_{j}}\right)\right)>\mu_{t}(\mathcal{E})
$$

Thus

$$
\mu_{\alpha}\left(\phi_{t_{j}}\right)+t_{j} \cdot \mu_{\beta}\left(\phi_{t}\right)>\mu_{\alpha}(\mathcal{E})+t_{j} \cdot \mu_{\beta}(\mathcal{E}) .
$$

When $j$ tends to $+\infty$, the inequality $(*)$ implies

$$
\lim \mu_{\alpha}\left(\phi_{t_{j}}\right) \geq \mu_{\alpha}(\mathcal{E}) \text {. }
$$

In fact, $\lim \mu_{\alpha}\left(\phi_{t_{j}}\right)$ exists (after possibly passing to "subsequences"), since $\mu_{\alpha}\left(\phi_{t_{j}}\right)<\mu_{\alpha}(\mathcal{E})$ and since $\mu_{\alpha}\left(\phi_{t_{j}}\right)$ is also bounded from below.

So we have

$$
\lim \mu_{\alpha}\left(\phi_{t_{j}}\right)=\lim \mu_{t_{j}}\left(\phi_{t_{j}}\right) \geq \mu_{\alpha}(\mathcal{E}) .
$$

We will obtain a contradiction to the $\alpha$-stability of $\mathcal{E}$, if we can show that $\mu_{\alpha}\left(\phi_{t_{j}}\right)$ takes only finitely many values. To verify that, notice that we already saw the existence of a positive constant $C$ such that

$$
0<\mu_{\alpha}\left(\phi_{t_{j}}\right) \leq C
$$

for $j$ sufficiently large (we may assume $\mu_{\alpha}(\mathcal{E})>0$ ).

Then we obtain-possibly after passing to a sequence converging to 0--a decomposition

$$
c_{1}\left(\phi_{t_{j}}\right)=A+B_{t_{j}}
$$

with $A$ and $B_{t}$ real classes such that $B_{t} \cdot \alpha=0$.

This decompostion comes from the following easy fact.

Let $f: \mathbb{R}^{n} \rightarrow \mathbb{R}$ be linear and non-zero be defined over $\mathbb{Q}$. Let $C>0$ and consider

$$
M=\left\{x \in \mathbb{R}^{n} \mid 0 \leq f(x) \leq C\right\} .
$$

Let $\left(a_{j}\right)$ be an infinite sequence in $M \cap \mathbb{Z}^{n}$. Then after passing possibly to a subsequence we have a decomposition

$$
a_{j}=b+c_{j}
$$

with $b, c_{j} \in M$ and $f\left(c_{j}\right)=0$.

Indeed: the map $f$, being defined over $\mathbb{Q}$, takes only finitely many values (depending on the denominators of the coefficients of $f$ ) on $M \cap \mathbb{Z}^{n}$.

From $(+)$ we obtain:

$$
\mu_{\alpha}\left(\phi_{t_{j}}\right)=\mu_{\alpha}(A)
$$

and the conclusion.

In Theorem 1.4 we made use of the following result of independent interest.

TOME $139-2011-\mathrm{N}^{\mathrm{O}} 1$ 
THEOREM 5.7. - Let $X$ be a projective manifold of dimension $n$ and $\pi: \tilde{X} \rightarrow$ $X$ a birational map from a projective manifold $\tilde{X}$. Let $H_{1}, \ldots, H_{n-1}$ be ample divisors on $\tilde{X}$ and set $\alpha=\pi_{*}\left(H_{1} \cdots H_{n-1}\right)$. Let $\mathcal{F}$ be an $\alpha$-semi-stable torsion-free sheaf on $X$. Let the suffix $p$ always denote reduction mod $p$. Then the sheaf $\mathcal{F}_{p}$ is $\alpha_{p}$-semi-stable for large $p$.

This result is well-known when $\pi$ is isomorphic, see e.g. [20], p.65.

Proof. - Suppose to the contrary that $\mathcal{F}_{p}$ is not $\alpha_{p}$-stable for infinitely many primes $p$ and let $\phi_{p} \subset \mathcal{F}_{p}$ be $\alpha_{p}$-destabilizing. The proof consists in bounding the Hilbert polynomials of the sheaves $\phi_{p}$, so that there exists a sheaf $\phi$ in characteristic 0 inducing the sheaves $\phi_{p}$. Since the slope with respect to $\alpha$ and $\alpha_{p}$ is always the same, $\phi$ destabilizes $\mathcal{F}$, a contradiction.

Let $Q_{p}=\mathcal{F}_{p} / \phi_{p}$. By Proposition 5.8 below, we may assume $\operatorname{rk}\left(Q_{p}\right)=1$, hence we can write $Q_{p}=\mathcal{I}_{Z_{p}} \otimes G_{p}$ with a line bundle $G_{p}$ and $Z_{p}$ of codimension at least 2. Since $G_{p}^{*}$ destabilizes $\mathcal{F}_{p}^{*}$, it suffices to bound $G_{p}$ instead of $\phi_{p}$. For this, it suffices to bound $\pi^{*}\left(G_{p}\right)=: \tilde{G}_{p}$.

Indeed: if $\tilde{\mathcal{F}}_{p}:=\pi^{*}(\mathcal{F}) /$ torsion, then $\tilde{G}_{p}$ might not be saturated in $\tilde{\mathcal{F}}_{p}^{*}$ any more. Thus our arguments below only show that $\mathscr{I}_{D_{p}} \otimes \tilde{G}_{p}$ is bounded for some effective divisor $D_{p}$; hence we obtain a line bundle $\hat{G}$ in char 0 such that $\hat{G}_{p}=I_{D_{p}} \otimes \tilde{G}_{p}$. But since $\operatorname{dim} \pi\left(D_{p}\right) \leq n-2$, we simply set $G=\pi_{*}(\hat{G})^{* *}$ and we are thus done. Hence we may, and shall assume from the beginning that $\tilde{G}_{p}$ is saturated in $\tilde{F}_{p}$.

To establish the boundedness of $\tilde{G}_{p}$ we shall show that the set of possible Hilbert polynomials

$$
P(m)=\chi\left(\tilde{X}, \tilde{G}_{p}+m H\right)
$$

is finite. Here we assume for simplicity that $H=H_{j}$ for all $j$ (in general consider the Hilbert polynomials for the various $H_{j}$ ). We may also assume that $H$ is very ample. Once this is done a relative Picard scheme argument finishes the proof.

Step 1. We bound $\left|c_{1}\left(\tilde{G}_{p}\right) \cdot h_{p}\right|$, where $h_{p}=H_{p}^{n-1}$.

Observe that, by the remarks before Lemma 5.3, we have:

$$
c_{1}\left(\tilde{G}_{p}\right) \cdot h_{p}=c_{1}\left(G_{p}\right) \cdot \alpha_{p}
$$

Therefore one part of the bound follows from the bound

$$
c_{1}\left(G_{p}\right) \cdot \alpha_{p} \leq C c_{1}\left(\mathcal{F}_{p}\right) \cdot \alpha_{p}
$$

with a constant $C$, using the fact that $G_{p}^{*}$ destabilizes $\mathcal{F}_{p}^{*}$. The other bound can be seen as follows: we may assume from the beginning that $\mathcal{F}$ is ample, hence $\mathcal{F}_{p}$ is ample, and therefore the generic quotient $G_{p}$ is big. Hence $c_{1}\left(G_{p}\right) \cdot \alpha>0$. 
Step 2. We now argue by induction on the dimension of $X$. We show that it suffices to bound $\chi\left(\tilde{G}_{p}\right)$. Choose $Y \in|H|$ general. By the induction hypothesis, $\tilde{G}_{p} \mid Y_{p}$ is bounded. Now

$$
\chi\left(\tilde{G}_{p}\left(m H_{p}\right)\right)=\chi\left(\tilde{G}_{p}\right)+\sum_{k=1}^{m} \chi\left(S, \tilde{G}_{p}\left(m H_{p}\right)\right),
$$

from which our claim already follows.

Step 3. We finally bound $\chi\left(\tilde{G}_{p}\right)$. We choose a positive integer $N$ such that

$$
\left(\tilde{G}_{p}-N H_{p}\right) \mid Y_{p}
$$

is negative for all $\tilde{G}_{p}$. This $N$ can be choosen independently on $\tilde{G}$, since the $\tilde{G}_{p} \mid Y_{p}$ are bounded, by the induction hypothesis. Then we obtain for all $N^{\prime} \geq N$ and all $q \leq n-1$ an embedding:

$$
H^{q}\left(\tilde{X}_{p}, \tilde{G}_{p}-N^{\prime} H_{p}\right) \rightarrow H^{q}\left(\tilde{X}_{p}, G_{p}-N H_{p}\right),
$$

and for $q \leq n-2$ the vanishing

$$
H^{q}\left(\tilde{X}_{p}, \tilde{G}_{p}-N^{\prime} H_{p}\right)=0
$$

Since

$$
h^{q}\left(\tilde{X}_{p}, \tilde{G}_{p}\right) \leq \sum_{i=1}^{N} h^{q}\left(Y_{p},\left(\tilde{G}_{p}-i H_{p}\right) \mid Y_{p}\right)+h^{q}\left(\tilde{X}_{p}, \tilde{G}_{p}-N H_{p}\right)
$$

we deduce the boundedness of $h^{q}\left(\tilde{X}_{p}, \tilde{G}_{p}\right)$ already for $q \leq n-2$.

In case $q=n$, we have

$$
h^{n}\left(\tilde{X}_{p}, \tilde{G}_{p}\right)=h^{0}\left(\tilde{X}_{p}, \tilde{G}_{p}^{*} \otimes K_{\tilde{X}_{p}}\right) \leq h^{0}\left(\tilde{X}_{p}, \mathcal{F}_{p}^{*} \otimes K_{\tilde{X}_{p}}\right) .
$$

It remains to consider the case $q=n-1$. We have an exact sequence

$$
0 \rightarrow \tilde{\phi}_{p} \rightarrow \tilde{\mathcal{F}}_{p} \rightarrow \mathscr{I}_{\tilde{Z}} \otimes \tilde{G}_{p} \rightarrow 0
$$

where $\tilde{Z}$ has codimension at least 2 . To bound $h^{n-1}\left(\tilde{X}_{p}, \tilde{G}_{p}\right)$ it suffices of course to bound $h^{n-1}\left(\tilde{X}_{p}, \tilde{\mathcal{J}}_{\tilde{Z}} \otimes \tilde{G}_{p}\right)$ and therefore to bound $h^{n}\left(\tilde{X}_{p}, \tilde{\mathscr{\phi}}_{p}\right)$ by taking cohomology of the last exact sequence. Dually, we need to bound $h^{0}\left(\tilde{X}_{p}, \tilde{\varnothing}^{*} \otimes\right.$ $\left.K_{\tilde{X}_{p}}\right)$. We substitute $\tilde{F}$ by $\tilde{F} \otimes m H$, where $m$ is so large that $\left(\tilde{\phi}_{p} \otimes m H_{p} \otimes\right.$ $\left.\left(-K_{\tilde{X}_{p}}\right)\right) \mid Y_{p}$ is ample for all $p$. This is possible since by induction we already have boundedness when restricting to $Y_{p}$. Therefore after the substitution, the sheaf $\left(\tilde{\phi}^{*} \otimes K_{\tilde{X}_{p}}\right) \mid Y_{p}$ is negative, the same being true for generic deformations of $Y_{p}$. Hence $H^{0}\left(\tilde{X}_{p}, \tilde{\varnothing}^{*} \otimes K_{\tilde{X}_{p}}\right)=0$, and we are done.

TOME $139-2011-\mathrm{N}^{\mathrm{O}} 1$ 
Proposition 5.8. - Let $X$ be a smooth projective variety over an algebraically closed field. Let $\mathcal{F}$ be a torsion free sheaf which is unstable w.r.t some movable class $\alpha$. Then $\Lambda^{r} \mathcal{F}$ (modulo torsion) is also $\alpha$-unstable for all $r<\operatorname{rk} \mathcal{F}$.

Proof. - Let $\& \subset \mathcal{F}$ be maximally destabilizing, introducing a sequence

$$
0 \rightarrow \varnothing \rightarrow \mathcal{F} \rightarrow Q \rightarrow 0
$$

with a torsion free sheaf $Q$. Since all computations will only invoke $c_{1}$, we may assume all three sheaves to be locally free. By arguing with $\mathbb{Q}$-bundles or by passing to symmetric powers, we may furthermore assume $c_{1}(\mathscr{F})=0$, in particular $c_{1}(\mathcal{F}) \cdot \alpha=0$ and hence $c_{1}(Q) \cdot \alpha<0$.

Let $m$ be the rank of $\mathcal{F}$.

If $r \leq m$, we obtain an epimorphism

$$
\bigwedge^{r} \mathcal{F} \rightarrow \bigwedge^{r} Q \rightarrow 0
$$

Since $c_{1}\left(\bigwedge^{r} \mathcal{F}\right) \cdot \alpha=0$ and since $c_{1}\left(\bigwedge^{r} Q\right) \cdot \alpha<0$, the bundle $\bigwedge^{r} \mathcal{F}$ is unstable in this case, too.

If $r>m$, we obtain an epimorphism

$$
\bigwedge^{r} \mathscr{F} \rightarrow \bigwedge^{m} Q \otimes \bigwedge^{r-m} \& \rightarrow 0
$$

Now an elementary calculation shows that

$$
c_{1}\left(\bigwedge^{m} Q \otimes \bigwedge^{r-m} \varnothing\right)=a c_{1}(Q) \cdot \alpha
$$

where

$$
a=\left(\begin{array}{l}
s-m \\
r-m
\end{array}\right)-\left(\begin{array}{l}
s-m-1 \\
r-m-1
\end{array}\right)>0 .
$$

Hence the conclusion is as before.

\section{Appendix: an analytic proof of Theorem 5.1 (by Matei Toma)}

We give here an analytic proof of Theorem 5.1. This proof needs no adaptation of the Grauert-Mülich Theorem. The main ingredient will be the Kobayashi-Hitchin correspondence for non-Kähler polarizations which was established by J. Li and S.T. Yau.

We shall use the notations and the definitions of the main paper. In particular $X$ will be a complex projective manifold of dimension $n \geq 2$. Following [7] we shall denote by $N_{\mathrm{amp}}$ the interior of the closed cone $\overline{M E(X)}$ generated by movable curves, see also [2]. It is easy to check using [2] that geometric classes of curves belong to $N_{\mathrm{amp}}$. 
The following proposition replaces Proposition 5.2.

Proposition 6.1. - Let $\alpha$ be a class in $N_{\mathrm{amp}}$ and $\mathcal{E}$ and $\mathcal{F}$ two $\alpha$-polystable locally free sheaves. Then $\mathcal{E} \otimes \mathcal{F}$ is again $\alpha$-polystable.

Proof. - We start by a Hahn-Banach argument and show the existence of a smooth positive definite form $u$ of bidegree $(n-1, n-1)$ with $\partial \bar{\partial} u=0$ and such that the slope of a holomorphic vector bundle with respect to $\alpha$ is computed by

$$
\mu_{\alpha}(E)=\frac{\int c_{1}(E, h) \wedge u}{\operatorname{rank} E},
$$

where $c_{1}(E, h)$ is the first Chern form of $E$ computed with respect to some hermitian metric $h$ in the fibers of $E$, cf. [7] Theorem 4.1.

Let indeed $\mathscr{D}_{1,1}^{+}$be the cone of positive currents inside the space $\mathscr{D}_{1,1}^{\prime}$ of currents of bidegree $(1,1)$. For any choice of a positive definite smooth $(1,1)$-form $\eta$ the set

$$
\mathscr{D}_{(1,1), \eta}^{+}=\left\{T \in \mathscr{D}_{1,1}^{+} \mid \int_{X} T \wedge \eta^{n-1}=1\right\}
$$

is compact for the weak topology on $\mathscr{D}_{1,1}^{\prime}$, see [6], III.1.23. The vector subspace

$$
V=\left\{T \in \mathscr{D}_{1,1}^{\prime} \mid \mathrm{d} T=0,[T] \cdot \alpha=0\right\}
$$

is closed and disjoint from $\mathscr{D}_{(1,1), \eta}^{+}$by the duality Theorem 2.4 in [2].

(Notice that $\alpha$ belongs also to the interior of the cone of movable classes $\left.\mu \subset H_{\mathbb{R}}^{n-1, n-1}(X)\right)$.

Thus there exists a continuous linear functional which is positive on $\mathscr{D}_{(1,1), \eta}^{+}$ and vanishes on $V$. This is given by a smooth positive definite form $u$ of bidegree $(n-1, n-1)$ which is also $\partial \bar{\partial}$-closed since $\partial \bar{\partial} \mathscr{D}_{0,0}^{\prime} \subset V$. Moreover a renormalization of $u$ by a positive factor makes $\alpha$ and $u$ to be equal as linear functionals on $H^{1,1}(X)_{\mathbb{R}}$ since they have the same kernel and are both positive on Kähler classes.

Next we take a $(n-1)$-st root $\omega$ of $u$ in the following way. First notice that

$$
\left(i \sum_{1 \leq i, j \leq n} a_{i j} \mathrm{~d} z_{i} \wedge \mathrm{d} \bar{z}_{j}\right)^{n-1}=(n-1) ! i^{(n-1)^{2}} \sum_{1 \leq i, j \leq n}(-1)^{i+j} c_{j i} \mathrm{~d} \hat{z}_{i} \wedge \mathrm{d} \hat{\bar{z}}_{j}
$$

where $c_{i j}$ denotes the cofactor of $a_{i j}$ in the matrix $A=\left(a_{i j}\right)_{1 \leq i, j \leq n}, \mathrm{~d} \hat{z}_{i}=$ $\mathrm{d} z_{1} \wedge \ldots \wedge \mathrm{d} z_{i-1} \wedge \mathrm{d} z_{i+1} \wedge \ldots \wedge \mathrm{d} z_{n}$ and $\mathrm{d} \hat{\bar{z}}_{j}=\mathrm{d} \bar{z}_{1} \wedge \ldots \wedge \mathrm{d} \bar{z}_{j-1} \wedge \mathrm{d} \bar{z}_{j+1} \wedge \ldots \wedge \mathrm{d} \bar{z}_{n}$. The relation ${ }^{t} C A=\operatorname{det}(A) I_{n}$ for the cofactor matrix $C=\left(c_{i j}\right)_{1 \leq i, j \leq n}$ implies

$$
A=\sqrt[n-1]{\operatorname{det}(C)^{t}} C^{-1}
$$

in case $A$ is positive definite. Moreover, given a positive definite matrix $C$, one obtains a unique positive definite solution $A$ of the equation (1). 
Then $\omega$ is the $(1,1)$-form associated to a Gauduchon metric on $X$ and

$$
\mu_{\alpha}(E)=\mu_{\omega}(E)=\frac{\int c_{1}(E, h) \wedge \omega^{n-1}}{\operatorname{rank} E}
$$

for $E$ and $h$ as before. By [15] the Kobayashi-Hitchin correspondence holds in this case, thus the polystability of a holomorphic vector bundle $E$ with respect to $\omega$ is equivalent to the existence of a Hermite-Einstein metric with respect to the polarization $\omega$ again. But the tensor product of Hermite-Einstein vector bundles is also Hermite-Einstein and the proposition is proved.

\section{BIBLIOGRAPHY}

[1] S. Boucksom - "Cônes positifs des variétés complexes compactes", thèse de doctorat, Université de Grenoble, 2002.

[2] S. Boucksom, J.-P. Demailly, M. Paun \& T. Peternell - "The pseudo-effective cone of a compact Kähler manifold and varieties of negative Kodaira dimension", to appear in J. Alg. Geom., 2011.

[3] F. CAMPANA - "Fundamental group and positivity of cotangent bundles of compact Kähler manifolds", J. Algebraic Geom. 4 (1995), p. 487-502.

[4] F. Campana, J. A. Chen \& T. Peternell - "Strictly nef divisors", Math. Ann. 342 (2008), p. 565-585.

[5] F. CAmpanA \& Q. ZHANG - "Compact Kähler threefolds of $\pi_{1}$-general type", in Recent progress in arithmetic and algebraic geometry, Contemp. Math., vol. 386, Amer. Math. Soc., 2005, p. 1-12.

[6] J.-P. Demailly - "Complex analytic and algebraic geometry", http:// WwW-fourier.ujf-grenoble.fr/ demailly/books.html.

[7] J.-P. Demailly, T. Peternell \& M. Schneider - "Holomorphic line bundles with partially vanishing cohomology", in Proceedings of the Hirzebruch 65 Conference on Algebraic Geometry (Ramat Gan, 1993), Israel Math. Conf. Proc., vol. 9, Bar-Ilan Univ., 1996, p. 165-198.

[8] H. Esnault - "Fibre de Milnor d'un cône sur une courbe plane singulière", Invent. Math. 68 (1982), p. 477-496.

[9] D. Huybrechts \& M. LeHN - The geometry of moduli spaces of sheaves, Aspects of Mathematics, E31, Friedr. Vieweg \& Sohn, 1997.

[10] Y. KaWAMATA - "Minimal models and the Kodaira dimension of algebraic fiber spaces", J. reine angew. Math. 363 (1985), p. 1-46.

[11] , "Pluricanonical systems on minimal algebraic varieties", Invent. Math. 79 (1985), p. 567-588. 
[12] _ "Moderate degenerations of algebraic surfaces", in Complex algebraic varieties (Bayreuth, 1990), Lecture Notes in Math., vol. 1507, Springer, 1992, p. 113-132.

[13] J. KOLLÁR - "Shafarevich maps and plurigenera of algebraic varieties", Invent. Math. 113 (1993), p. 177-215.

[14] A. LANGER - "Semistable sheaves in positive characteristic", Ann. of Math. 159 (2004), p. 251-276.

[15] J. LI \& S.-T. YAU - "Hermitian-Yang-Mills connection on non-Kähler manifolds", in Mathematical aspects of string theory (San Diego, Calif., 1986), Adv. Ser. Math. Phys., vol. 1, World Sci. Publishing, 1987, p. 560573.

[16] V. B. Mehta \& A. Ramanathan - "Semistable sheaves on projective varieties and their restriction to curves", Math. Ann. 258 (1981/82), p. 213224.

[17] Y. MiYAoKA - "Deformations of a morphism along a foliation and applications", in Algebraic geometry, Bowdoin, 1985 (Brunswick, Maine, 1985), Proc. Sympos. Pure Math., vol. 46, Amer. Math. Soc., 1987, p. 245-268.

[18] _ "Relative deformations of morphisms and applications to fibre spaces", Comment. Math. Univ. St. Paul. 42 (1993), p. 1-7.

[19] Y. MiYaoka \& S. Mori - "A numerical criterion for uniruledness", Ann. of Math. 124 (1986), p. 65-69.

[20] Y. Miyaoka \& T. Peternell - Geometry of higher-dimensional algebraic varieties, DMV Seminar, vol. 26, Birkhäuser, 1997.

[21] C. Mourougane - "Théorèmes d'annulation générique pour les fibrés vectoriels semi-négatifs", Bull. Soc. Math. France 127 (1999), p. 115-133.

[22] Y. Namikawa \& J. H. M. SteenbrinK - "Global smoothing of CalabiYau threefolds", Invent. Math. 122 (1995), p. 403-419.

[23] N. I. Shepherd-BARRon - "Miyaoka's theorem on the seminegativity of $T_{X}$ ", Astérisque 211 (1992), p. 103-114.

[24] _ _ "Semi-stability and reduction $\bmod p "$, Topology 37 (1998), p. 659664.

[25] C. Simpson - "Subspaces of moduli spaces of rank one local systems", Ann. Sci. École Norm. Sup. 26 (1993), p. 361-401.

[26] E. VIEHWEG - "Weak positivity and the additivity of the Kodaira dimension for certain fibre spaces", in Algebraic varieties and analytic varieties (Tokyo, 1981), Adv. Stud. Pure Math., vol. 1, North-Holland, 1983, p. 329-353. 